This document is confidential and is proprietary to the American Chemical Society and its authors. Do not copy or disclose without written permission. If you have received this item in error, notify the sender and delete all copies.

\title{
Atomistic Details of Chymotrypsin Conformational Changes upon Adsorption on Silica
}

\begin{tabular}{|c|c|}
\hline Journal: & ACS Biomaterials Science \& Engineering \\
\hline Manuscript ID & $a b-2018-00819 m \cdot R 1$ \\
\hline Manuscript Type: & Article \\
\hline $\begin{array}{r}\text { Date Submitted by the } \\
\text { Author: }\end{array}$ & 26-Sep-2018 \\
\hline Complete List of Authors: & $\begin{array}{l}\text { Hildebrand, Nils; Hybrid Materials Interfaces Group, Faculty of Production } \\
\text { Engineering, Bremen Center for Computational Materials Science } \\
\text { (BCCMS) and Center for Environmental Research and Sustainable } \\
\text { Technology (UFT) } \\
\text { Michaelis, Monika; Hybrid Materials Interfaces Group, Faculty of } \\
\text { Production Engineering, Bremen Center for Computational Materials } \\
\text { Science (BCCMS) and Center for Environmental Research and } \\
\text { Sustainable Technology (UFT) } \\
\text { Wurzler, Nina; Hybrid Materials Interfaces Group, Faculty of Production } \\
\text { Engineering, Bremen Center for Computational Materials Science } \\
\text { (BCCMS) and Center for Environmental Research and Sustainable } \\
\text { Technology (UFT) } \\
\text { Li, Zhuo; University of Nottingham, School of Chemistry } \\
\text { Hirst, Jonathan; University of Nottingham, School of Chemistry } \\
\text { Micsonai, Andr\&aacute;s; Eötvös Loránd University, Department of } \\
\text { Biochemistry } \\
\text { Kardos, József; Eötvös Loránd University, Department of Biochemistry } \\
\text { Gil-Ley, Alejandro; Scuola Internazionale Superiore di Studi Avanzati, } \\
\text { Statistical and Biological Physics } \\
\text { Bussi, Giovanni; Scuola Internazionale Superiore di Studi Avanzati, } \\
\text { Statistical and Biological Physics } \\
\text { Köppen, Susan; Hybrid Materials Interfaces Group, Faculty of Production } \\
\text { Engineering, Bremen Center for Computational Materials Science } \\
\text { (BCCMS) and Center for Environmental Research and Sustainable } \\
\text { Technology (UFT) } \\
\text { Delle Piane, Massimo; Hybrid Materials Interfaces Group, Faculty of } \\
\text { Production Engineering, Bremen Center for Computational Materials } \\
\text { Science (BCCMS) and Center for Environmental Research and } \\
\text { Sustainable Technology (UFT) } \\
\text { Colombi Ciacchi, Lucio; Hybrid Materials Interfaces Group, Faculty of } \\
\text { Production Engineering, Bremen Center for Computational Materials } \\
\text { Science (BCCMS) and Center for Environmental Research and } \\
\text { Sustainable Technology (UFT) }\end{array}$ \\
\hline
\end{tabular}

\section{SCHOLARONE" Manuscripts}




\title{
Atomistic Details of Chymotrypsin
}

\section{Conformational Changes upon Adsorption on}

\section{Silica}

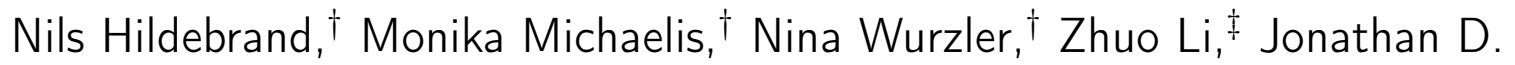 \\ Hirst, ${ }^{\ddagger}$ András Micsonai, ${ }^{\Uparrow}$ József Kardos, $₫$ Alejandro Gil-Ley, ${ }^{\S}$ Giovanni Bussi, ${ }^{\S}$ \\ Susan Köppen, ${ }^{\dagger}$ Massimo Delle Piane, ${ }^{*}{ }^{\dagger}$ and Lucio Colombi Ciacchi ${ }^{\dagger}$ \\ $\dagger$ Hybrid Materials Interfaces Group, University of Bremen, Faculty of Production \\ Engineering, Bremen Center for Computational Materials Science, Center for \\ Environmental Research and Sustainable Technology (UFT), and MAPEX Center for \\ Materials and Processes, Am Fallturm 1, 28359 Bremen, Germany \\ $\ddagger$ School of Chemistry, University of Nottingham, University Park, Nottingham NG7 2RD, \\ United Kingdom \\ \ELTE Eötvös Loránd University, Pázmány Péter sétány 1/C, Budapest, H-1117 Hungary \\ §International School for Advanced Studies (SISSA), via Bonomea 265, 34136 Trieste, Italy \\ E-mail: massimo.dellepiane@hmi.uni-bremen.de
}

\begin{abstract}
Adsorption of enzymes on solid surfaces may lead to conformational changes that reduce their catalytic conversion activity and are thus detrimental to the efficiency of biotechnology or biosensing applications. This work is a joint theoretical and experimental endeavor in which we identify and quantify the conformational changes that


chymotrypsin undergoes when in contact with the surface of amorphous silica nanoparticles. For this purpose, we use circular dichroism spectroscopy, standard molecular dynamics and advanced-sampling methods. Only the combination of these techniques allowed us to pinpoint a destabilization effect of silica on specific structural motifs of chymotrypsin. They are linked by the possibility of theoretically predicting CD spectra, allowing us to elucidate the source of the experimentally observed spectral changes. We find that chymotrypsin loses part of its helical content upon adsorption, with minor perturbation of its overall tertiary structure, associated to changes in the aromatic interactions. We demonstrate that the C-terminal helical fragment is unfolded as an isolated oligopeptide in pure water, folded as an $\alpha$-helix as terminus of chymotrypsin in solution, and again partly disordered when the protein is adsorbed on silica. We believe that the joint methodology introduced in this manuscript has a direct general applicability to investigate any biomolecule - inorganic surface system. Methods to theoretically predict Circular Dichroism spectra from atomistic simulations were compared and improved. The drawbacks of the approaches are discussed; in particular the limited capability of advanced-sampling MD schemes to explore the conformational phase space of large proteins, and the dependency of the predicted ellipticity bands on the choice of calculation parameters.

\section{Keywords}

Protein adsorption, Silica, Circular dichroism, Molecular dynamics, Free energy, Conformational changes

\section{Introduction}

Interactions at the abiotic/biotic interface have become of eminent interest in fields ranging from basic research, pharmaceutical formulation development, up to industrial product de- 
sign, leaving several open challenges. ${ }^{112}$ The issue of biomolecules adsorption onto inorganic surfaces in a wet environment is of great importance for the design and functionalization of biomaterials in the context of biomedical implants, biosensors, drug delivery and antifouling surfaces. $\frac{3+5}{5}$ Enzymes, antibodies and nucleic acids combined with inorganic substrates, for instance silica nanoparticles, are increasingly proposed for therapeutic and in vivo applications. ${ }^{6}$ Furthermore, protein-based drugs are often stored in glass vials, particularly when they are solvated in aqueous solutions. Unwanted adsorption at the vials' walls can restrict the shelf life of the formulation due to concentration variations, changes in activity and evolution of undesirable effects. ${ }^{[7}$ Additionally, the functionalization of materials surfaces with enzymes have found a wide range of applications in biotechnology and biosensing, for instance to impart catalytic or antimicrobial properties to otherwise inert materials. $\frac{89}{8}$

Proteins might undergo changes in their conformation (denaturation) upon interaction with a solid material surface, which can detrimentally affect their biological or enzymatic activity. ${ }^{10}$ How these changes can be predicted, described and correlated to physico-chemical features of the material is however still unclear. Protein denaturation is commonly induced by adsorption on hydrophobic surfaces, whereas hydrophilic surfaces in general better preserve the native protein structures. $\frac{11-15}{15}$ However, even small conformational changes can reduce the enzymatic activity, as frequently observed on functionalized oxide nanoparticles of alumina, titania or silica. $\stackrel{16] 17}{ }$ The present work aims at giving a precise, atomic-level insight into the conformational changes that a well-known enzyme (chymotrypsin) experiences when it adsorbs on the surface of a widely employed oxide, amorphous silica $\left(\mathrm{SiO}_{2}\right)$.

In previous studies $\frac{18 \mid 19}{19}$ we have shown that chymotrypsin adsorbs on silica in a very well defined orientation, namely with its two helical regions facing the surface. There, it attaches strongly via the anchoring action especially of its positively charged and neutral polar residues, which exhibit high affinity towards the surface's terminal groups, in particular the deprotonated silanols. Here we investigate whether and to what extent this anchoring promotes a structural change in the helical regions in contact with the surface. Other authors 
have indeed reported a loss of the helical structure upon adsorption of chymotrypsin on $\mathrm{SiO}_{2}$, as inferred from Circular Dichroism (CD) experiments. $\stackrel{20}{20}$ On the other hand, adsorption seems to not significantly affect the tertiary structure of chymotrypsin. 21

The C-terminal $\alpha$-helix of chymotrypsin may be prone to unfolding after interaction with silica ${ }^{18}$ Helices can change their conformation dramatically when moving from a bulk solution to a solid/liquid interface. For instance, the C-terminal fragment of lysozyme is helical in the native protein, assumes a random-coil structure when put alone in aqueous solvent, and recovers the original helical fold after immersion in trifluoroethanol. ${ }^{22} \mathrm{~A}$ fragment of collagen XIV assumes a predominantly random conformation in pure water or adsorbed at a hydrophilic silicon/water interface. ${ }^{23}$ However, adsorption at hydrophobic organic surfaces ${ }^{24}$ or on hydrogen-terminated silicon ${ }^{23}$ results in stabilization of an $\alpha$-helical conformation. Other studies have investigated the stabilization of helical motifs after adsorption on either hydrophobic $\frac{25}{25}$ or hydrophilic $\stackrel{2627}{27}$ surfaces. These effects are strongly dependent on the surface affinity of the sequence of individual amino acid residues. For instance, the wellstudied 4DAR5 peptide is helical in aqueous solution, but readily unfolds after adsorption on silica. 28229

Experimental investigation of the secondary structure of a protein in an adsorbed state is not trivial, and CD spectroscopy is one of the few techniques that has been successfully

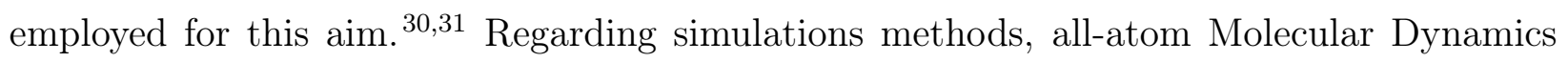
(MD) based on classical force fields may be very promising, given its proven ability to predict the dynamics of biomolecular systems with high precision. However, standard MD simulations access time scales typically of the order of $100 \mathrm{~ns}$ to $1 \mu \mathrm{s}$, which is not sufficient to overcome potential energy barriers associated with conformational changes taking place over seconds to hours. For example, chymotrypsin adsorbed on silica did not show any substantive conformational change in MD simulations of $300 \mathrm{~ns}$ at room temperature. $\frac{18}{\text { So }}$ called advanced-sampling MD methods are required to capture the rare events leading to conformational changes in proteins and improve the ergodicity of the simulation. $\frac{32[33}{3}$ 
Replica Exchange with Solute Tempering (REST) in combination with MetaDynamics $(\text { MetaD })^{34}$ is an advanced-sampling MD method that has been successfully used to predict the conformational ensemble of oligopeptides in solution or adsorbed on materials sur-

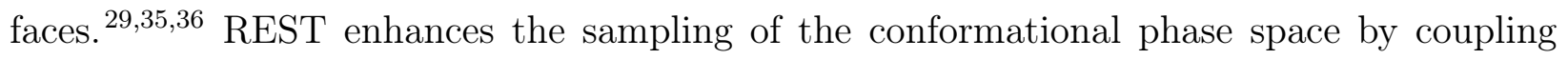
the pristine system to a fictitious set of replicas at higher temperatures. The temperature rescaling (tempering) can be applied only to a portion of the system (the "solute"), to avoid dealing with the very large number of degrees of freedom of its surrounding environment (the "solvent"). $\stackrel{33137}{37}$ Metadynamics, on the other hand, ensures accurate sampling along a pre-defined reaction coordinate, or Collective Variable (CV), by accumulating repulsive bias potentials along this coordinate while the system dynamically evolves. $\frac{3438}{38}$ The combination of both methods is very effective, as it leads to accurate profiles of the system's free energy along the CV, while ensuring that the orthogonal degrees of freedom are explored with sufficient accuracy thanks to the enhanced sampling of the high-temperature replicas. In this way, the biased evolution of the lower-temperature replicas provide accurate refinement of the predicted free-energy profiles, whereas the higher-temperature replicas ensure that energy barriers, acting on a priori unknown degrees of freedom, are easily overcome.

In this paper we complement the application of RESTmetaD with CD experiments to quantify the partial unfolding of chymotrypsin after adsorption on amorphous $\mathrm{SiO}_{2}$ nanoparticles. $\mathrm{SiO}_{2}$ (especially Stöber silica) is particularly suitable as an adsorption material in CD experiments because its refractive index (1.42) is very similar to that of water (1.33), so that light scattering does not significantly affect the measurements. As a result, CD analysis of a suspension of silica nanoparticles with proteins adsorbed on their surfaces provides only protein-specific information, thus giving the possibility of estimating the amount of secondary structure in the adsorbed state. An important link between the experimentally measured spectra and the simulations can be made via the theoretical calculation of the CD spectra based on the atomic positions of the sampled trajectory by using a dedicated web interface such as Dichrocalc ${ }^{39}$ or calculate the CD spectra from the secondary structure com- 


\section{Materials and Methods}

\section{Circular dichroism (CD) experiments}

CD experiments were performed with $\alpha$-chymotrypsin from bovine pancreas purchased from Sigma-Aldrich (Purity $\geq 85 \%$, LOT:110M7009V) and an oligopeptide with sequence RVTALVNWVQQTLAAN, corresponding to the chymotrypsin's helical C-terminal fragment, synthezised by JPT (purity $\geq 95 \%$ Berlin, Germany). The as-purchased protein was dissolved in $\mathrm{ddH}_{2} \mathrm{O}(18 \mathrm{M} \Omega$, MilliQ, Synergy, Millipore, Germany) at a stock concentration of $20 \mathrm{mg} \mathrm{ml}^{-1}$, as quantified by absorbance at 205 and $214 \mathrm{~nm} .{ }^{4142}$ The as-purchased oligopeptide was dissolved in $\mathrm{ddH}_{2} \mathrm{O}$ at a concentration of $1 \mathrm{mg} \mathrm{ml}^{-1}$, as quantified by absorbance at 205 and $214 \mathrm{~nm}$.

CD spectra were recorded with an Applied Photophysics Chirascan spectrometer running the Pro-Data Chirascan software (v4.2.22). At least three repeat scans for each sample were measured at $25^{\circ} \mathrm{C}$, over the wavelength range of 190 to $250 \mathrm{~nm}$ using intervals of $1 \mathrm{~nm}$, in Suprasil quartz cells (Hellma UK Ltd.) with pathlengths ranging from 0.001 to $0.1 \mathrm{~cm}$. The scans were averaged, the respective baseline subtracted and the resulting net spectra smoothed with a Savitsky-Golay Filter using smoothing windows of 5 to 10 data points.

The mean residue ellipticity $\left(\Theta_{M R E}\right)$ was defined as ${ }^{43}$

$$
\Theta_{M R E}=\frac{\Theta}{c \cdot l \cdot n}
$$

where $\Theta$ is the raw $\mathrm{CD}$ ellipticity, $n$ is the number of amino acids in the solvated protein or peptide, $l$ is the pathlength of the used quartz cuvette and $c$ the concentration of the protein. 
To estimate the contribution of specific secondary structure components in the samples, the

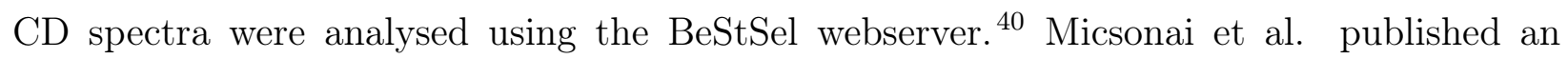
extended set of eight secondary structure components that have contribution to the CD spectra with special emphasis on the different $\beta$-sheet elements defined by different twists. $\underline{40}$

The temperature ramping experiments were performed at a protein concentration of $1 \mathrm{mg} \mathrm{ml}^{-1}$ in $\mathrm{ddH}_{2} \mathrm{O}$ and a pathlength of $0.1 \mathrm{~cm}$, using the stepped ramp mode starting from $25^{\circ} \mathrm{C}$ and increasing the temperatures in steps of $5^{\circ} \mathrm{C}$ with a heating time of 3 min per step. At every step, three spectra were collected and averaged.

The fumed silica nanoparticles for the adsorption study were purchased from SigmaAldrich (purity $\geq 99.8 \%$, LOT: SLBG4255V), with a BET surface area of $192.4 \mathrm{~m}^{2} \mathrm{~g}^{-1}$ (Belsorp min, BEL Japan, Inc.). The zeta-potential and hydrodynamic diameter in $\mathrm{ddH}_{2} \mathrm{O}$ were determined with a Delsa Nano C Particle Analyzer (Beckman Coulter, US). The nanoparticles showed a zeta potential of $-32 \pm 2 \mathrm{mV}$ and a hydrodynamic diameter of $189 \pm 9 \mathrm{~nm}$. Prior to each experiment, the nanoparticle suspension was treated with an ultrasonic homogenisator (Sonoplus HD 3400, Amplitude 50 \%, Pulse 0.5 s, Bandelin, Berlin, Germany) for $5 \mathrm{~min}$, to prevent agglomeration. A CD measurement of the concentration of silica nanoparticles is performed prior the adsorption study to obtain a baseline, which allows for a removal of their absorbance influence on the measurements of the protein.

CD spectra of $\alpha$-chymotryspin adsorbed on amorphous silica were collected after incubation of the protein stock solution in a suspension containing $10 \mathrm{mg} \mathrm{ml}^{-1}$ of silica nanoparticles, leading to a protein concentration of $10 \mathrm{mg} \mathrm{ml}^{-1}$. After an incubation time of $24 \mathrm{~h}$ in an overhead shaker at $30 \mathrm{rpm}$ (Loopster digital, IKA, Germany) the suspension was centrifuged at $14000 \mathrm{~g}$ for $10 \mathrm{~min}$ (Microcentrifuge 1814, VWR, Germany). The supernatant was removed completely and analyzed with respect to its absorbance at 205 and $214 \mathrm{~nm}$ as well as its CD spectrum. Additionally, $\alpha$-chymotrypsin was incubated for $24 \mathrm{~h}$ in an overhead shaker and the CD spectrum and the concentration were analyzed to ensure that potential spectral changes were not related to degradation of the protein or interaction with the reaction vial. 
The adsorbance analysis was used to determine the concentration of chymotrypsin remaining in solution ( $80 \%$ of the starting concentration) and, by subtraction from the original amount, the concentration of adsorbed chymotrypsin (20\% of the starting concentration) (c.f. S.I., Fig.S1)

The pellet was resuspended in the same amount of $\mathrm{dd}_{2} \mathrm{O}$ as previously removed, and CD spectra were collected in the shortest possible time (three spectra within less than 5 min), to avoid as much as possible the desorption of the adsorbed proteins from the nanoparticles. The baseline obtained from the nanoparticle suspension is subtracted from the measured CD spectrum to remove the contribution of the nanoparticles. The as-determined ellipticities were converted to $\Theta_{M R E}$ values based on the determined respective concentrations, allowing for their direct comparison before and after adsorption. We performed a second centrifugation step following the measurement of the re-suspended nanoparticles with the adsorbed proteins after waiting for $1 \mathrm{~h}$. The CD measurement on the corresponding supernatant enable us to determine the protein desorbed from the nanoparticles (c.f. S.I., Fig.S1). We find that a maximum of $50 \%$ of the protein detaches in comparison to the adsorbed concentration.

We note that the procedure is not completely free from possible uncertainties caused by the potential partial desorption of proteins after resuspension and the adsorbance from the $\mathrm{SiO}_{2}$ nanoparticles themselves, despite the care taken to ensure that the reference samples (baselines) contained the same amount of silica as the chymotrypsin-containing samples.

\section{Atomistic models for chymotrypsin and silica}

The $\alpha$-chymotrypsin structure was taken from the Protein Data Bank archive (PDB entry: 4CHA). The two missing residues, GLY12 and LEU13, were inserted to the sequence using the LEAP tool included in the AMBER simulation package. ${ }^{44}$ The protonation states of ASP, GLU, HIS were adjusted employing the constant-pH simulation method ${ }^{44}$ to a neutral $\mathrm{pH}$, which results in a protein net charge of +3 e (histidine protonation state: HIS38: HIE; HIS55: HID). The resulting protein model is shown in Fig. 1. In addition to whole chymotrypsin 


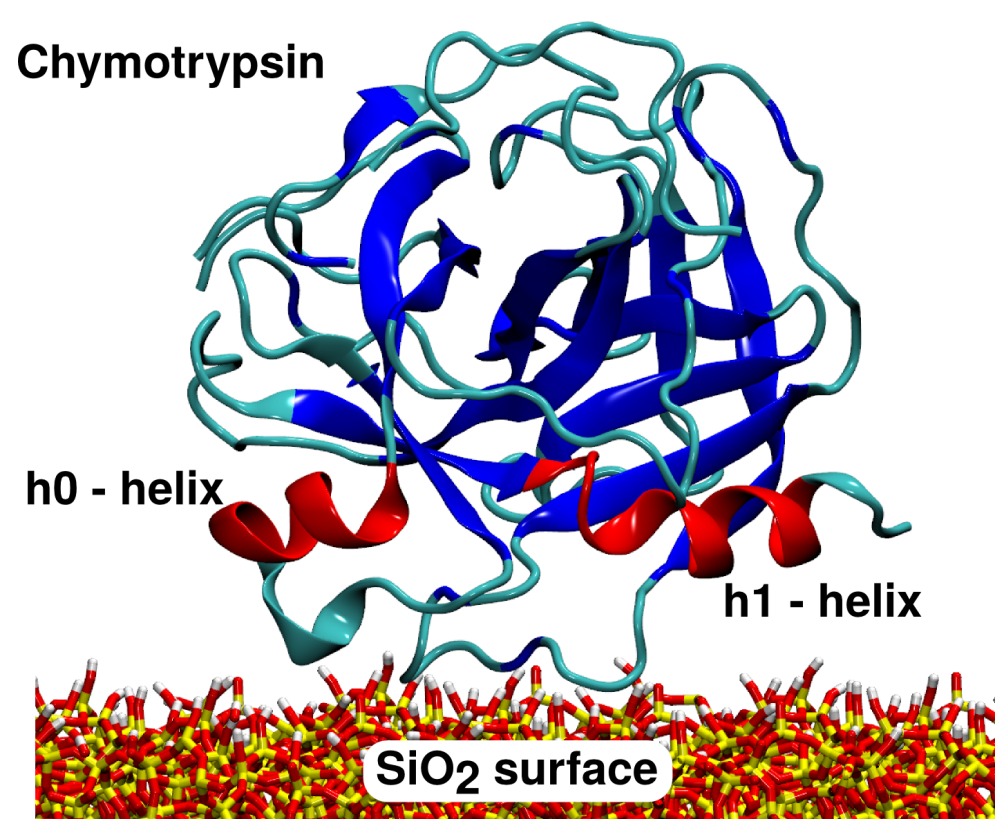

Figure 1: Schematic representation of chymotrypsin in close contact with the silica surface model (Si, yellow; O, red; H, white). The two $h_{0}$ and $h_{1}$ helices are highlighted in red. The $\beta$-sheets are colored in blue, while the other secondary structure components are visualized in cyan.

we have also simulated its isolated C-terminal fragment with sequence RVTALVNWVQQTLAAN, corresponding to residue numbers 230 to 245, at the same protonation conditions.

As in previous work, $\frac{18 \mid 19}{12}$ the structure of the amorphous $\mathrm{SiO}_{2}$ surface was taken from Cole et al. 45 The surface slab, lying in the $x y$ plane of the periodically repeated simulation box, had a thickness of $21 \AA$ and an area of $90 \times 90 \AA^{2}$. The surface slab was separated from its periodically repeated image along the $z$ coordinate perpendicular to the $x y$ plane by about $74 \AA$, resulting in a free volume above the surface which was filled with water molecules and chymotrypsin. This surface has a silanol surface density of $4.4 \mathrm{OH} \mathrm{nm}^{-2}$. The surface charge was adjusted by deprotonating a number of silanol terminal groups up to a surface charge of $-0.07 \mathrm{C} \mathrm{m}^{-2}$, in line with several potentiometric titration experiments. $\underline{46}+\underline{49}$ A surface segment is included in Fig. 1. 


\section{Molecular Dynamics (MD) simulations}

All MD simulations were performed with GROMACS,, 50 employing the AMBER14SB force field ${ }^{44}$ in combination with the ionsjc_tip3p force field for the ions ${ }^{51}$ and the force field of Butenuth et al. ${ }^{52}$ for the $\mathrm{SiO}_{2} /$ water interface. Lorentz-Berthelot combination rules were applied to the van der Waals parameters. Bonds involving hydrogen atoms were constrained by means of the LINCS algorithm. ${ }^{53}$ All surface atoms except the silanol hydrogens were kept fixed in their equilibrium positions. The simulation box used to model the dissolved RVTALVNWVQQTLAAN peptide (with net charge $+1 e$ ) included $6558 \mathrm{H}_{2} \mathrm{O}$ molecules and $1 \mathrm{Cl}^{-}$ion to ensure charge neutrality. The simulation box used for dissolved chymotrypsin included $21337 \mathrm{H}_{2} \mathrm{O}$ molecules and three $\mathrm{Cl}^{-}$ions. The simulation box used for chymotryspin interacting with the $\mathrm{SiO}_{2}$ surface included $21071 \mathrm{H}_{2} \mathrm{O}$ molecules and $69 \mathrm{Na}^{+}$counterions. The Particle Mesh Ewald (PME) method was used for the calculation of the electrostatic interactions, using a cutoff distance of $1.2 \mathrm{~nm}$ for the real-space part of the Ewald sums and the van der Waals interactions.

In all systems, the solvent density was adjusted to the equilibrium TIP3P water density at $300 \mathrm{~K}$ and $1 \mathrm{~atm}$, i.e. $0.983 \pm 0.001 \mathrm{~g} \mathrm{~cm}^{-3} .18$ Prior to the production runs, the protein and peptide were equilibrated in a series of NVT, NPT and NVE runs for about $1 \mathrm{~ns}$, according to standard protocols. Constant-temperature simulations were performed in an NVT ensemble with a modified Berendsen thermostat with a coupling constant of $0.1 \mathrm{ps} . \stackrel{54}{ }$ Keeping the volume constant was necessary to avoid instabilities of the simulation cell caused by the high water temperature. Since the simulations at temperatures higher than $300 \mathrm{~K}$ were used only to obtain qualitative information about the unfolding behaviour of chymotrypsin, the resulting increase of pressure was not significant for the interpretation of the results. A Verlet integration time step of $2 \mathrm{fs}$ was used. Visualization and analysis of the trajectories were performed with VMD. $\underline{55}$ 


\section{Advanced-sampling molecular dynamics simulations}

Free energy profiles associated with the helical unfolding of chymotrypsin were computed with Replica Exchange with Solute Tempering (REST) $)^{33137 / 56}$ combined with Metadynamics (MetaD),,$\sqrt[34]{ }$ as employed in previous works. ${ }^{29 \mid 36}$ We focused on the change of conformation of the C-terminal fragment $\mathrm{h}_{1}$ (residues 230-245), which is helical in the equilibrium structure of chymotrypsin in solution (colored red in the scheme in Fig. 2).

Solely this part of the system was defined as the 'solute' in the REST simulations, whose temperature was scaled in the different system replicas ('hot' system region). The rest of the protein, the water and the salt ions remained at the base temperature $T_{0}=300 \mathrm{~K}$ ('cold' system region). We used 12 replicas at temperatures $T_{i}$ corresponding to 300.0, 324.0, 350.0, 378.0, 408.3, 440.9, 476.3, 514.4, 555.6, 600.1, 648.1, and $700 \mathrm{~K}$, respectively. Defining $\beta_{i}=1 /\left(k_{\mathrm{B}} T_{i}\right)$, where $k_{\mathrm{B}}$ is the Boltzmann constant, the Lennard-Jones parameters $\epsilon$ of the hot atoms in the $i$-th replica were scaled by the factor $\beta_{i} / \beta_{0}$, and their charges $q$ by

the factor $\sqrt{\beta_{i} / \beta_{0}}$. Of the bonded interactions, only the dihedral force constants $k_{\psi}$ were scaled. If both distal atoms of a dihedral bond (atoms 1 and 4 in Fig. 2 belonged to the hot region, the scaling factor was $\beta_{i} / \beta_{0}$. When one atom was hot and the other cold, the scaling factor was $\sqrt{\beta_{i} / \beta_{0}} \cdot \frac{37}{}$ Exchanges between the replicas were attempted every 400 fs, following a Metropolis-Hastings acceptance criterion. The geometric progression of the temperatures $T_{i}$ ensured a nearly uniform overlap of the potential energy distributions and thus a uniform acceptance probability across the replica ladder, $\frac{57}{5 i t h}$ average values between $46 \%$ and $48 \%$ in the simulated systems. The round-trip time, which is defined as the time needed by one replica to move along the complete temperature ladder from 300 to $700 \mathrm{~K}$ and back, amounted to $0.48 \pm 0.04 \mathrm{~ns}$ for the dissolved oligopeptide, $0.77 \pm 0.84 \mathrm{~ns}$ for the dissolved protein and $0.51 \pm 0.12 \mathrm{~ns}$ for the protein/surface system.

Further conformational sampling was achieved by means of the 'well-tempered' meta-

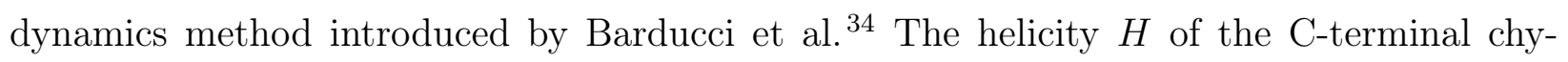
motrypsin fragment was used as the collective variable $(\mathrm{CV})$. This was defined, as in PLUMED 1, 
with $N$ corresponding to the total number of amino acids in the helix $(N=16$ for helix $\mathrm{h}_{1}$ and $N=8$ for helix $\mathrm{h}_{2}$ ) and the index $i$ spanning between residue numbers 231-244 (14 residues) and 166-171 (6 residues) for the helices $h_{1}$ and $h_{2}$, respectively. An ideal helix assumes a value $H=100 \%$, whereas a completely unordered structure assumes a value $H$ $=0 \%$. We have implemented this CV in PLUMED 2 by using the matheval library. ${ }^{59}$ The starting configuration of all MetaD simulations was chosen as the one with $H=0$. Control simulations were also run starting from opposite initial conditions $(H=100 \%$, S.I., Fig. S2) to check that the same free energy profile was obtained. Along the CV, Gaussian hills with width of $0.35 \mathrm{rad}$ were deposited with a frequency of $1 \mathrm{ps}^{-1}$. The initial height of the Gaussians, added every $\mathrm{N}=300$ steps, in each $i$-th REST replica was $h_{i}=\left(k_{\mathrm{B}} \Delta T_{i} / \tau\right) N \Delta t, \underline{60}$ where $\Delta t$ is the simulation time step ( $2 \mathrm{fs}$ ), $\tau$ is the characteristic time for the bias evolution in the well-tempered scheme ( $3 \mathrm{ps})$, and $\Delta T_{i}$ is the boosting temperature. The latter was defined as $\Delta T_{i}=T_{i}\left(\gamma_{i}-1\right),{ }^{38160}$ with bias factors $\gamma_{i}$ equal to $4,4.6,5.4,6.2,7.2,8.3,9.6,11.1$, $12.9,14.9,17.3,20$ in the 12 replicas, respectively. This resulted in boosting temperatures along the $\mathrm{CV}$ ranging from 900 to $5700 \mathrm{~K}$.

\section{Structural cluster analysis}

Cluster analysis of the structures present in the RESTmetaD trajectories was performed using the GROMOS algorithm, 61 according to the differences in the root-mean square displacement (RMSD) values of individual frames, with an RMSD cutoff of $0.3 \mathrm{~nm}$. The helix peptide in selected ranges of helicity $H$ (Eq.22) was aligned by a rigid rotation and translation of the whole system to minimize the RMSD variation prior to clustering. 
Non bonded

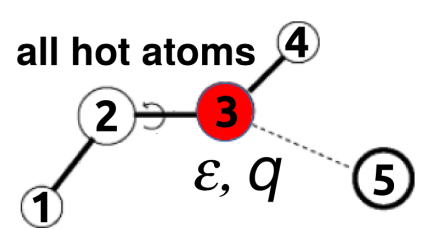

Bonded

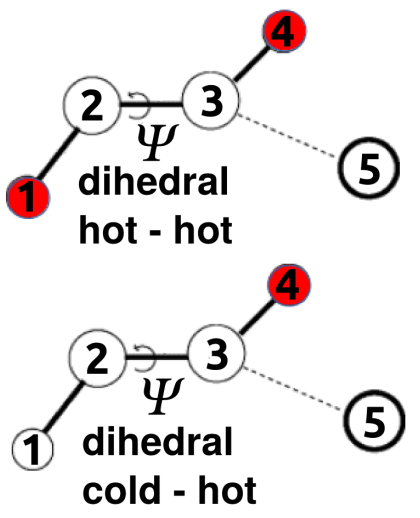

Interactions

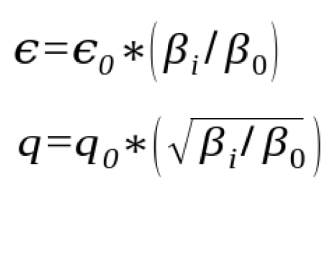

$k_{\psi}=k_{\psi, 0} *\left(\beta_{i} / \beta_{0}\right)$

$k_{\psi}=k_{\psi, 0} *\left(\sqrt{\beta_{i} / \beta_{0}}\right)$ $\mathrm{cc} \rightarrow$ cold-cold

hh $\rightarrow$ hot-hot

ch $\rightarrow$ cold-hot

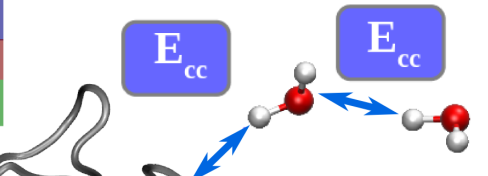

Figure 2: Parameter scaling of a solute tempering simulation of one protein part following the REST2 approach. (left) Schematic of four bonded atoms (1-4) and one non-bonded atom (5). Atoms defined as "hot" are red. The Lennard-Jones parameter $\epsilon$ as well as the charge $q$ of the "hot" atoms are scaled by the presented formula. The dihedral interactions are scaled differently if both or just one distal atom is "hot". (right) The C-terminus helix of chymotrypsin is defined as "hot" (red). Interactions are separated into cold-cold, hot-hot and hot-cold interaction types. $\beta_{i}=1 /\left(k_{\mathrm{B}} T_{i}\right)$.

\section{Calculation of theoretical CD spectra}

Theoretical CD spectra were calculated using the DichroCalc web interface ${ }^{39}$ and the BeStSel $\operatorname{method}^{40}$ for individual frames of the simulated trajectories. The calculation with DichroCalc involves the construction of an exciton Hamiltonian matrix, which models how electronic transitions interact with one another; $; 62$ the sign and magnitude of the interactions is dictated by the relative orientation and separation of chromophores, i.e., by the precise structure of the protein. The matrix elements were computed using three different parameter sets modeling the $n \rightarrow \pi^{*}$ and $\pi_{n b} \rightarrow \pi^{*}$ transitions of the peptide bond chromophore, namely: a set derived from semi-empirical calculations, $\frac{63}{6}$ a set based on ab initio calculations, $\frac{64}{6}$ and another $a b$ initio set in which the vibrational structure of the $\pi_{n b} \rightarrow \pi^{*}$ transition is explicitly included. $\frac{65}{6}$ The vibrational structure, arising from the Franck Condon progression of the 


\section{De novo secondary structure prediction}

To investigate the intrinsic conformational propensities of the C-terminal fragment RVTALVNWVQQTLAAN based on its primary structure, we applied three different secondary structure prediction servers. The PEP-Fold server predicts first Structural Alphabet (SA) 
letters from the sequence using a hidden Markov model approach. These fragments are assembled by a Greedy procedure driven by a modified OPEP coarse-grained force field energy score followed by a clustering procedure leading to a maximum of five 3D structures. $\frac{6768}{6}$ The iterative threading assembly refinement server (I-Tasser) is a hierarchical approach, which identifies structural templates from the PDB database. The full-length atomic models are constructed by iterative template fragment assembly using Replica Exchange Monte Carlo simulations. $\frac{69}{6}$ The QUARK prediction tool is a protein folding algorithm based on a Replica Exchange Monte Carlo simulation under the guide of an atomic-level knowledge-based force field. $\frac{70}{7}$ The major difference in comparison to the two other approaches is the prediction without homologous templates. The 16 mer peptide sequence required an extension via a GGGG linker since the minimum number of amino acids is 20 for the usage of this server.

\section{Results}

\section{Circular dichroism experiments}

We first report the experimentally measured CD spectra of chymotrypsin in solution, adsorbed on $\mathrm{SiO}_{2}$ nanoparticles, and subjected to a temperature increase leading to partial denaturation in solution (Fig. 3). The CD spectrum of native chymotrypsin is characterized by two distinct minima at about 205 and $230 \mathrm{~nm}$, separated by a rather flat region with a barely noticeable shoulder at $215 \mathrm{~nm} .20$ The minimum at $205 \mathrm{~nm}$ is associated with the $\pi \rightarrow \pi^{*}$ transition. ${ }^{71}$ The one at $230 \mathrm{~nm}$, on the other hand, has been tentatively assigned to the coupling of aromatic residues, namely Trp-Trp couplings or to Trp coupling with Phe. ${ }^{72}$ Adsorption of chymotrypsin on silica leads to disappearance of the minimum at $230 \mathrm{~nm}$ and a red shift of the minimum at $205 \mathrm{~nm}$, which also moves towards smaller absolute values of ellipticity. The disappearance of the $230 \mathrm{~nm}$ band suggests an enhanced flexibility of peptide chains around aromatic residues (Trp, Phe) causing a destabilization of their mutual interactions. ${ }^{74}$ A slight overall decrease of the signal intensity is also visible, 
but not quantifiable due to the uncertainty associated with the precise determination of the concentration of adsorbed proteins (see Methods). Analysis of the spectra via the BeStSel webserver ${ }^{40}$ revealed a $26 \%$ decrease of the protein's helicity (from $10.0 \pm 1.9 \%$ to $7.4 \pm$ $2.3 \%$ ) upon adsorption. The amount of parallel $\beta$-sheets is also slightly decreased, while the number of residues in unordered structures ('others' in the tables in Fig. 3) correspondingly increases. The CD spectra collected from the supernatant after re-desorption experiments reveal that these adsorption-induced structural changes are fully reversible (c.f. S.I., Fig.S1, panel (e)).
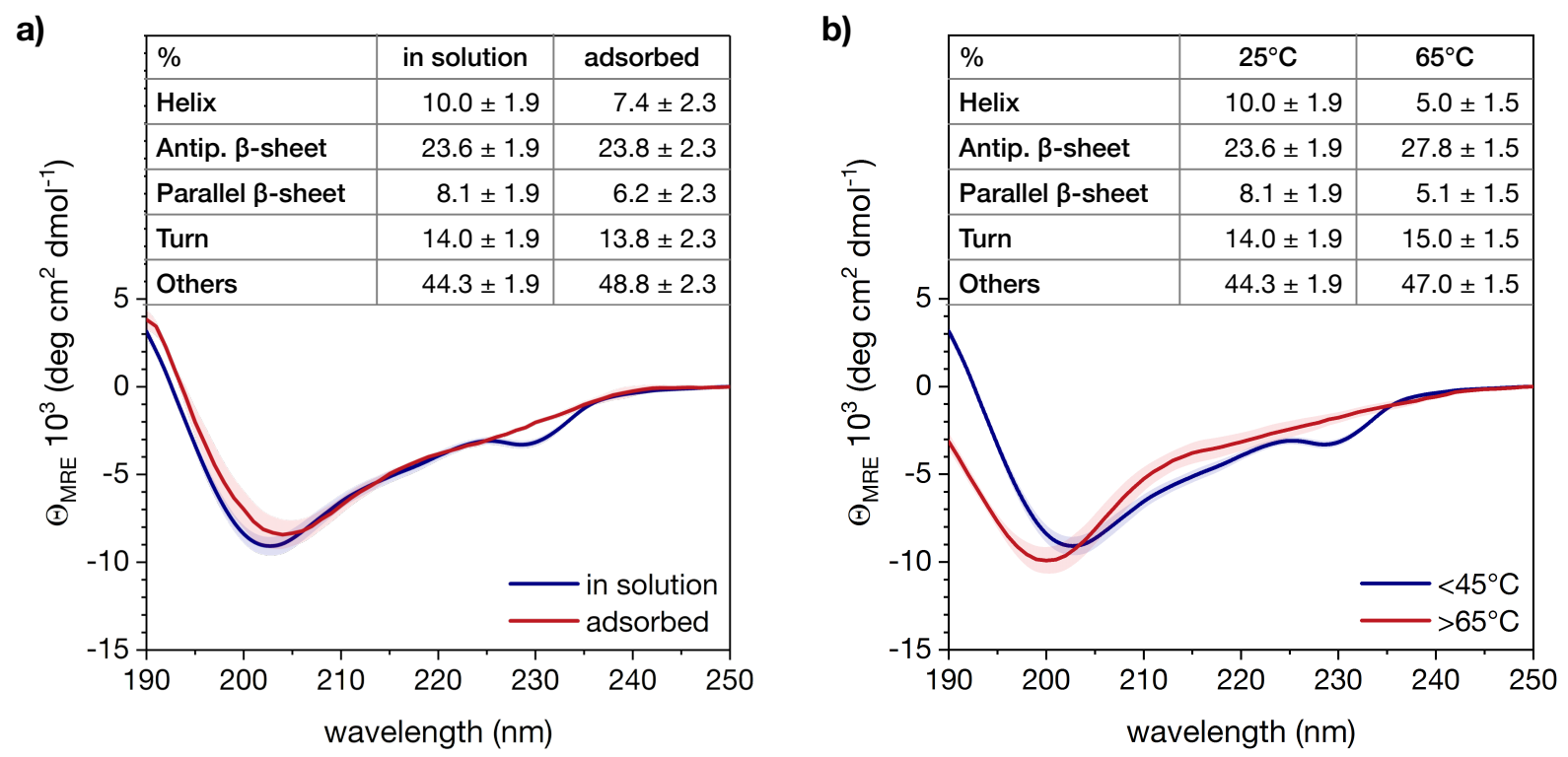

Figure 3: CD spectra of chymotrypsin dissolved in $d_{d H_{2}} \mathrm{O}$ and adsorbed on $\mathrm{SiO}_{2}$ nanoparticles (a); and dissolved in $d d \mathrm{H}_{2} \mathrm{O}$ subjected to a temperature ramping from $25^{\circ} \mathrm{C}$ to $65^{\circ} \mathrm{C}(\mathrm{b})$. The amount of secondary structure elements obtained by spectral analysis is reported in the inserts.

Heating the dissolved protein results in a slightly different behavior. Namely, in agreement with a previous study, 20 the minimum at $205 \mathrm{~nm}$ undergoes a blue shift, accompanied by an increase of the absolute ellipticity value in the region from 190 to $205 \mathrm{~nm}$. However, also in this case the minimum at $230 \mathrm{~nm}$ disappears (Fig. 3(b)), suggesting similar influence on the aromatic residues. At $65^{\circ} \mathrm{C}$, the helicity is reduced by $50 \%$. Other structural changes involve an enrichment in antiparallel and a depletion in parallel $\beta$-sheets, as well 
as an increase of the disordered structures. The spectral changes obtained from the heating procedure are found to be irreversible on the timescale of the experiment (see Supplementary Information, Fig.S1, panel (g)).

We note that the determination of secondary structure contributions from the CD spectra is strongly dependent on the method applied and the associated basis sets. $\frac{40}{\text { The basis }}$ sets are developed based on CD spectra with a known associated structure from XRD or NMR measurements. However, the application of an extended parameter set considering the spectral variability of structures associated with $\beta$-sheets within the BeStSel approach reduces the deviations in the calculation of secondary structure components for proteins $4 \underline{40}$ and peptides $\frac{75}{7 n}$ comparison to other approaches.

Partial loss of helicity due to either adsorption or heating is intriguing, given that in a previous study we have predicted that chymotrypsin adsorbs on silica in an orientation which brings both its helical fragments $\left(h_{0}\right.$ and $h_{1}$, see Fig. 1$)$ in contact with the surface. Therefore, silica could perturb the protein's native structure inducing partial helical unfolding. Since the $\mathrm{h}_{1}$ helix is located at the C-terminus, it is also conceivable that its structure would easily be altered at high temperature, which would be consistent with our structural analysis. In order to shed light on these issues, in the following we perform a detailed MD investigation, first on the basis of simple trajectories at high temperatures, and later making use of advancedsampling methods accessing the whole free-energy landscape of the protein.

\section{Standard molecular dynamics simulations at high temperature}

A total of eight standard MD simulations were performed with the protein either in solution or adsorbed on $\mathrm{SiO}_{2}$, at simulated temperatures of $300 \mathrm{~K}, 400 \mathrm{~K}, 500 \mathrm{~K}$ and $600 \mathrm{~K}$. Simulations were preceded by a $50 \mathrm{~ns}$ temperature ramping and equilibration. Temperatures higher than $300 \mathrm{~K}$ were used as a simple way to overcome local energy barriers and observe protein denaturation within a short simulation time. ${ }^{[76}$ In Fig. 4 we report the evolution of the helicity of the two helical fragments $\mathrm{h}_{0}$ (residues 165 to 172 in the chymotrypsin sequence) and 

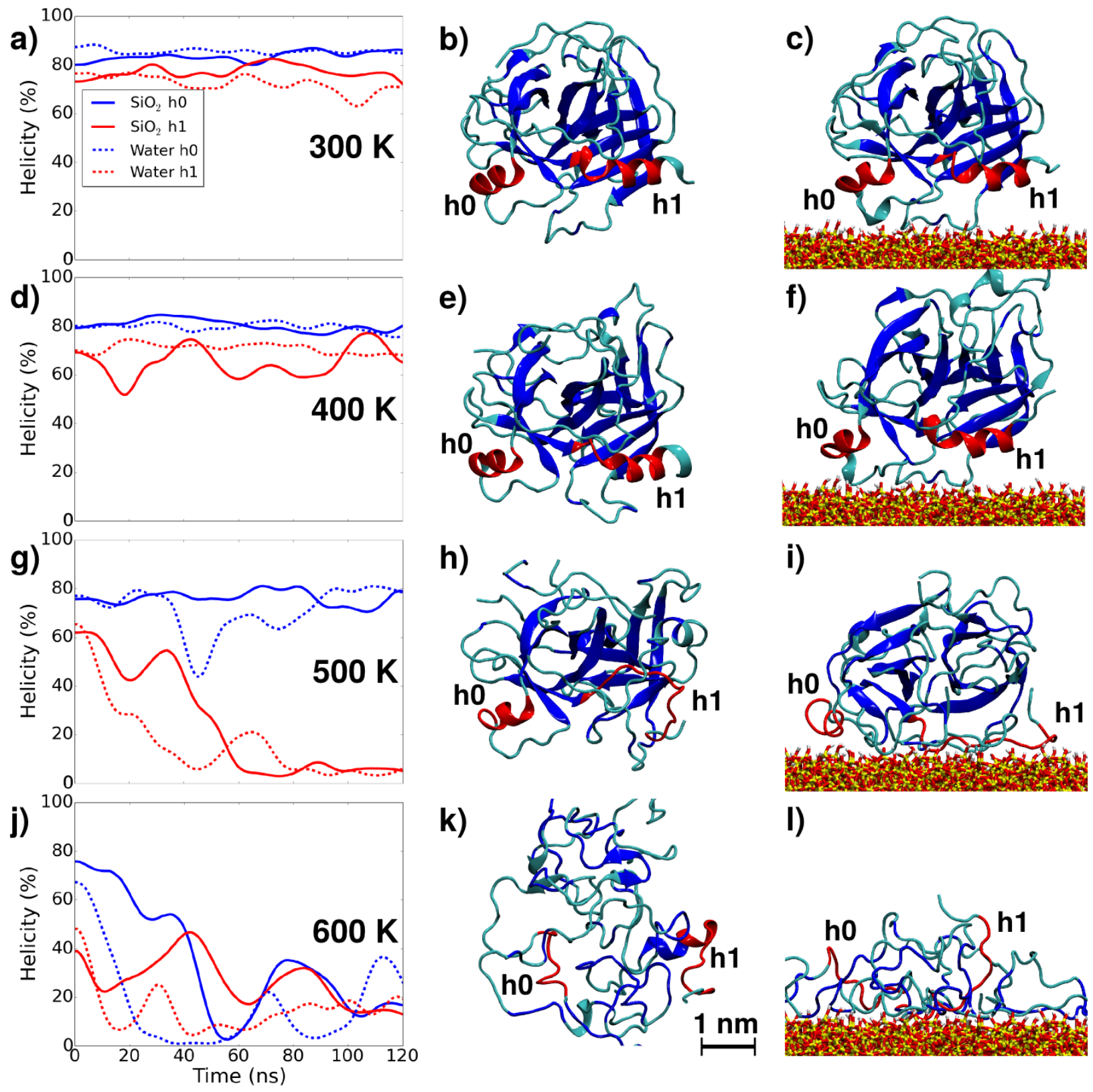

I)

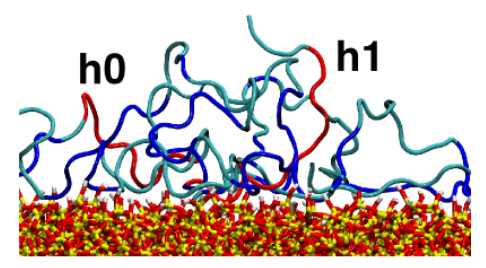

Figure 4: Results of standard MD simulations of chymotrypsin dissolved in water and adsorbed on the $\mathrm{SiO}_{2}$ surface at $300 \mathrm{~K}, 400 \mathrm{~K}, 500 \mathrm{~K}$ and $600 \mathrm{~K}$. The evolution of the helicity in the two helix regions $h_{0}$ and $h_{1}$ is shown in $(a, d, g, j)$ together with the final snapshots after 120 ns in water (b, $e, h, k)$ and on the surface $(c, f, i, l)$. The helicity is reported as percentage of the ideal $H$ value for $\alpha$-helices computed via Eq. 2. Simulations were preceded by a 50 ns temperature ramping and equilibration. Smoothing on the curves has been applied for clarity. 
$\mathrm{h}_{1}$ (residues 230 to 245), computed via Eq. 2, together with snapshots of the final structures after 120 ns of MD.

No obvious conformational changes can be observed during the simulations at $300 \mathrm{~K}$ and $400 \mathrm{~K}$, in which the helicity of both $\mathrm{h}_{0}$ and $\mathrm{h}_{1}$ remains close to the ideal values for $\alpha$-helices. On the contrary, at the highest temperature, $600 \mathrm{~K}$, severe unfolding within about 20 to $40 \mathrm{~ns}$ takes place both in water and on the surface during the simulations. More interesting is the behavior observed at $500 \mathrm{~K}$, where only the C-terminal helix $\mathrm{h}_{1}$ unfolds after about $50 \mathrm{~ns}$, whereas the other helix $\mathrm{h}_{0}$ maintains $80 \%$ of its helicity during the whole simulation. This behavior of helicity, as defined in Eq. 2, with temperature matches the behavior observed by looking at a more general secondary structure assignment via the STRIDE server $\frac{77}{\text { (S.I., }}$ Fig. S3).

These results suggest that heating of chymotrypsin is likely to induce unfolding of its C-terminal helix fragment before more severe denaturation takes place, which is consistent with the experimental CD analysis above (Fig. 3). Interestingly, preferential conformational changes at the C-terminus upon adsorption have been also reported in literature for the case of RNase A on $\mathrm{COOH}$ - terminated self-assembled monolayers and tentatively explained by electrostatic repulsion. ${ }^{78}$ Secondary structure variations in terminal residues have also been reported for other proteins in interaction with charged surfaces. ${ }^{79}$ Furthermore, analysis of the trajectories revealed a high sensitivity of some aromatic residues (Trp, Phe), close to the helical segments, to the observed conformational destabilization (S.I., Fig. S4). Namely, Trp-Trp and Trp-Phe couplings are lost or undergo rearrangements at $500 \mathrm{~K}$ (both in solution and adsorbed), which could explain the loss of the $230 \mathrm{~nm}$ minimum observed in experiments (Fig. 3). On the other hand, the aromatic residues located in the protein's $\beta$-sheet core are unaffected.

In these simulations no qualitative difference is observed in the presence or absence of the silica surface, apart from the fact that the $\mathrm{h}_{1}$ fragment does seem to adsorb on the surface when it unfolds (Fig. 4(i)). Before addressing whether surface-induced unfolding can actually 
take place, in the following we study the structural stability of the C-terminal fragment in more detail and the influence of its unfolding on theoretically predicted CD spectra.

\section{Calculation of CD spectra during helical unfolding}

CD spectra of proteins can be computed from first principles by means of exciton theory, in which the coupling of electronic excitations of individual chromophores is modelled through electrostatic interactions calculated using approximations of transition charge densities, e.g., sets of charges. Electronic transitions of the protein are, thus, linear combinations of the transitions of the isolated chromophores. This is the approach implemented in the Dichro-

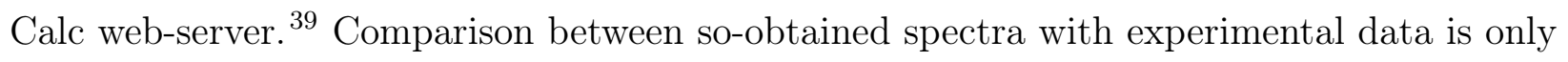
reliable under the condition that the considered structure is a very deep minimum of the energy landscape associated with possible conformational changes of the molecule. Otherwise, the spectra of all accessible structural conformations need to be computed and weighted by

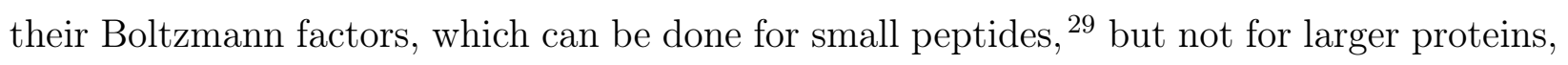
due to the excessive computational time required.

Alternatively, CD spectra of proteins can also be predicted through a statistics-based approach, by a superposition of a basis set of spectra associated with elementary secondary structure elements (helices, sheets, coils, etc.), weighted according to the amounts of those elements present in the protein structure, which needs to be determined a priori. This second approach can be applied using the basis spectra of e.g. the BeStSel method. $\stackrel{40}{\text { Given }}$ a certain molecular structure, its related CD spectrum can be theoretically predicted.

In this work, we generated a set of snapshots of the entire chymotrypsin forcing the unfolding of the $\mathrm{h}_{1}$ helix by means of a variable constraint associated with its helicity, as defined in Eq. 2, during an MD simulation at 300 K. For each of those snapshots we computed theoretical CD spectra using both DichroCalc with three different parameter sets and BeStSel with two different approaches, as described in Section 2.6. Namely, either by calculating the spectra directly from the BeStSel basis (that reflects low aromatic contents), 

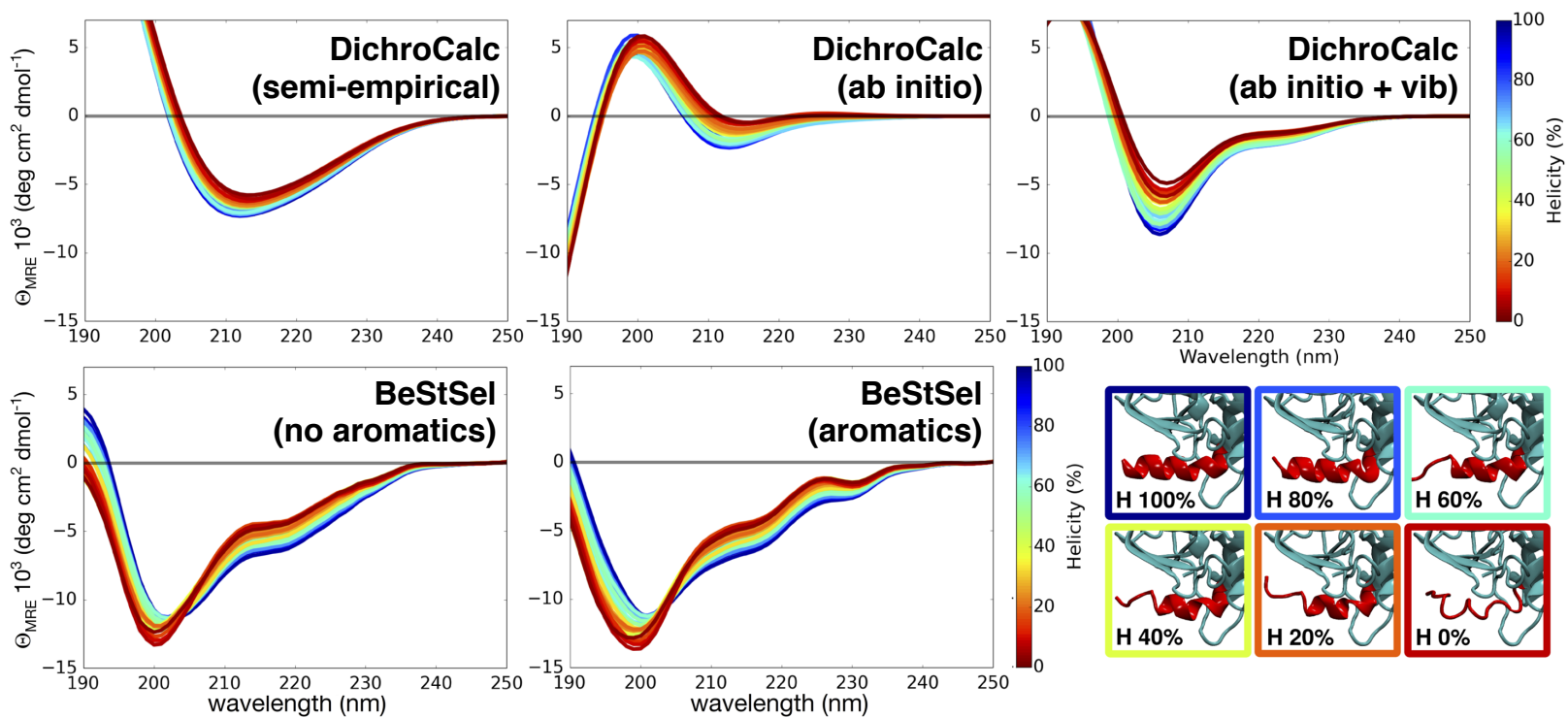

Figure 5: MD simulation at $300 \mathrm{~K}$ of chymotrypsin with a variable constraint applied to the helicity $H$ of the $h_{1}$ helix, which is forced to unfold from an ideal helix $(H=100 \%)$ to a fully unordered structure $(H=0 \%)$ over $30 \mathrm{~ns}$. The theoretically predicted CD spectra associated with 100 selected snapshots along the trajectory are reported, computed with the DichroCalc server using three parameter sets (semi-empirical, ab initio, ab initio + vib) and with the BeStSel server using two different basis sets (without and with aromatic contributions). See for the details. Selected snapshots of the $h_{1}$ structure (red ribbons) are shown in the bottom right.

or calculating the spectral deviation from the secondary structure change with respect to the native structure and subtracting it from the CD spectrum of the native protein, which better accounts for the the native aromatic contributions. We note, however, that in none of these methods is the true aromatic coupling between side-chain residues properly taken into account, which limits the theoretical CD analysis. The results are presented in Fig. 5.

Evident is the large variability of spectra predicted by the different methods and parameters or basis sets employed. Notably, the variability observed when changing the fraction of helical residues from $0 \%$ to $100 \%$ is less than that observed in the experiment. For native chymotrypsin (blue curves in the spectra of Fig. 5), all methods reproduce the deep minimum at about $205 \mathrm{~nm}$, although shifted towards $210 \mathrm{~nm}$ in some cases (semi-empirical, ab initio in Fig. 5).

With DichroCalc, only the parameter set including vibrational transitions (ab initio + vib in Fig. 5 is able to reproduce a second minimum, although at about $225 \mathrm{~nm}$ and not 
$230 \mathrm{~nm}$, and much less pronounced than in the experiments. During the unfolding process (blue to red in the spectra of Fig. 5), all DichroCalc parameter sets predict a red shift and a decrease in intensity for the $205 \mathrm{~nm}$ minimum, which is consistent with the changes in the experimental spectra (Fig. 3) induced by adsorption on silica.

BeStSel, without consideration of aromatic contributions, shows for $H=100 \%$ a minimum at $205 \mathrm{~nm}$ and a shoulder at about $220 \mathrm{~nm}$, which is roughly in correspondence with the experimentally observed shoulder between the deeper minima (see Fig. 3). With decreasing helicity, it predicts a blue shift and an increase of the intensity for the $205 \mathrm{~nm}$ minimum, while a decrease in intensity in the $210-240 \mathrm{~nm}$ region, which is the behavior observed in the experiments during chymotryspin heating. Upon consideration of aromatic contributions, the BeStSel spectra are enriched by a minimum at $230 \mathrm{~nm}$, in agreement with the experiment. The fact that this component is not present when the spectrum is calculated only from the pure BeStSel basis spectra, that consider only weak aromating contributions, is supporting the hypothesis regarding the influence of aromatic couplings. $\frac{72}{74}$ This minimum is conserved at all helicity values, at variance with what observed in the experiments during heating or adsorption (see Fig. 3), further indicating that it cannot be assigned to a secondary structure component. However, the analysis of the aromatic residues in proximity of the helical segments during the constrained MD simulation (S.I., Fig. S4) shows no variation in the Trp-Trp and Trp-Phe couplings between $H=100 \%$ and $H=0 \%$.

These predictions are therefore not entirely conclusive regarding the true conformational changes responsible for the different experimental spectra. For this reason, in the following we undergo a more thorough investigation using advanced-sampling methods that give access to the full free-energy profile associated with the helical unfolding process.

\section{Advanced-sampling simulations}

In order to investigate the stability of the C-terminal fragment $\mathrm{h}_{1}$, we first perform an analysis of the structure of the isolated hexadecapeptide fragment with sequence RVTALVN- 

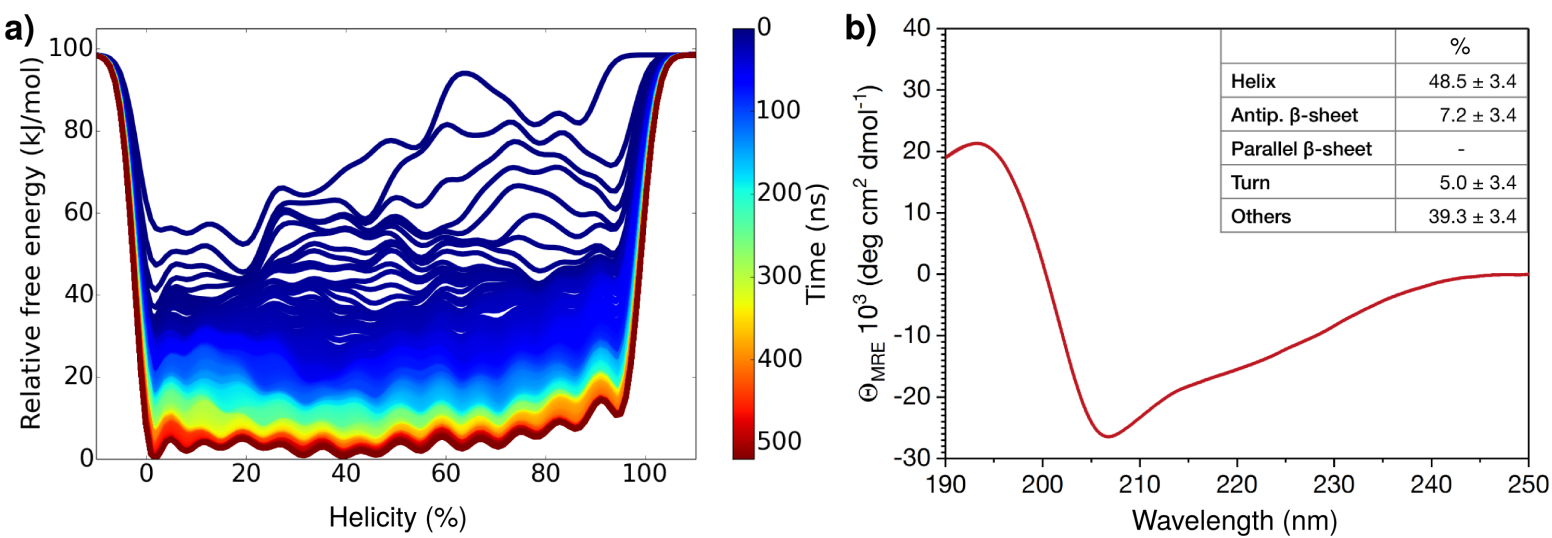

Figure 6: RESTmetaD free energy landscape (a) and experimental CD analysis (b) of the structure of the isolated C-terminal fragment of chymoptrypsin (residues 230 to 245, sequence RVTALVNWVQQTLAAN) in pure water.

WVQQTLAAN. Various secondary structure prediction servers predict for this sequence a predominantly, but not entirely, helical structure. For instance, the QUARK ${ }^{70}$ and I-Tasser ${ }^{69}$ tools suggest a helical conformation for $75.0 \%$ to $87.5 \%$ of the peptide; PEP-Fold ${ }^{[6768}$ predicts a $56.3 \%$ propensity for an $\alpha$-helical conformation.

We computed the free-energy profile associated with the folding/unfolding of the hexadecapeptide in pure water using RESTmetaD with the peptide's helicity (Eq. 2) as the collective variable. The time dependence of the estimated free energy profile over a $500 \mathrm{~ns}$ RESTmetaD simulation is shown in Fig. 6(a). The converged profile is very shallow across the entire range of helicity, with a series of 13 local minima (with depths of about $3 \mathrm{~kJ} \mathrm{~mol}^{-1}$ ) corresponding to the individual unfolding of the amino acid residues, as obtained before for a similar system. $\frac{29}{29}$ The global minimum is located at $H=0 \%$, but a secondary shallow minimum only about $1 \mathrm{~kJ} \mathrm{~mol}^{-1}$ higher is present at $H$ values between $30 \%$ and $50 \%$. Boltzmann integration of the entire profile predicts an average value of helicity $H=36.7 \%$.

In order to support this finding with experimental evidence, we measured the CD spectrum of the isolated RVTALVNWVQQTLAAN peptide in $\mathrm{ddH}_{2} \mathrm{O}$ and analysed its secondary structure via the BeStSel server (Fig. 6(b)). The analysis reveals a helical content slightly smaller than $50 \%$, which is in fair agreement with the results of the prediction servers and 

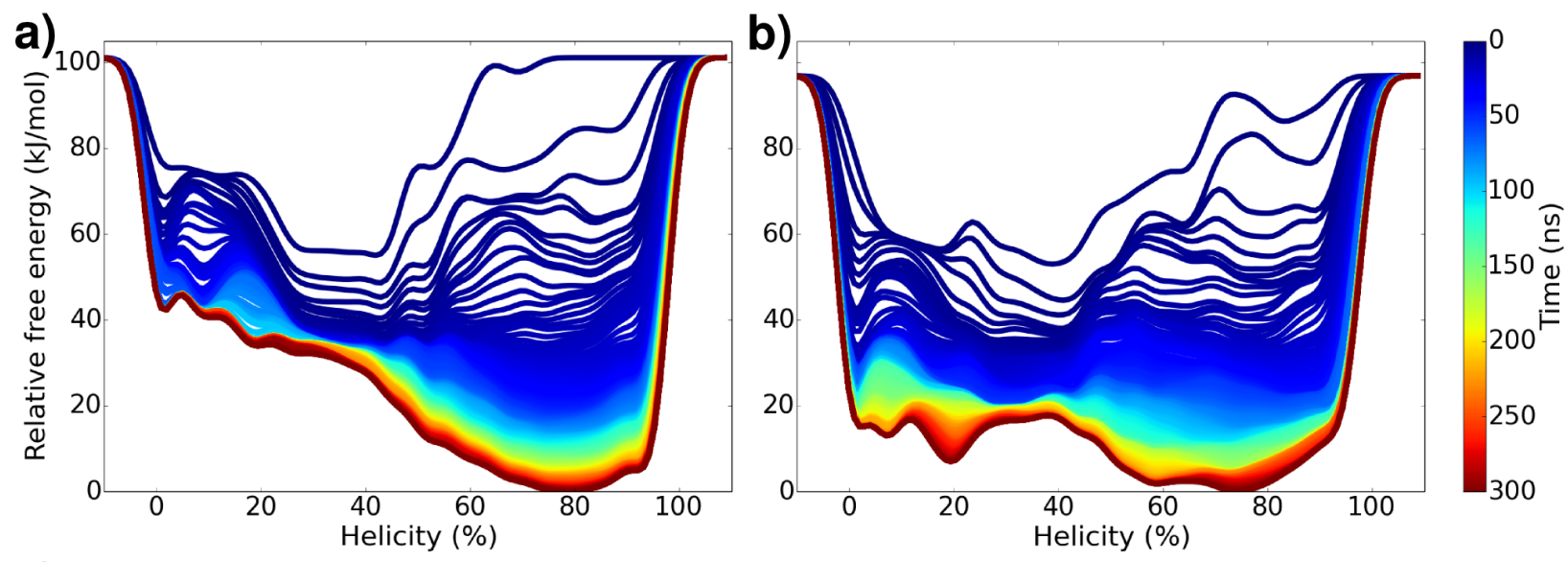

c)

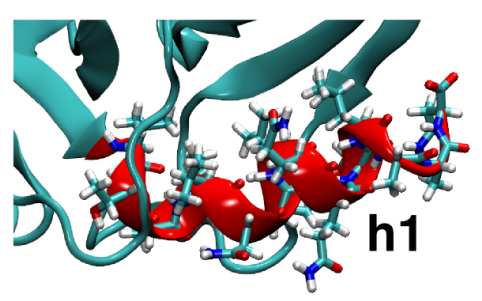

d)

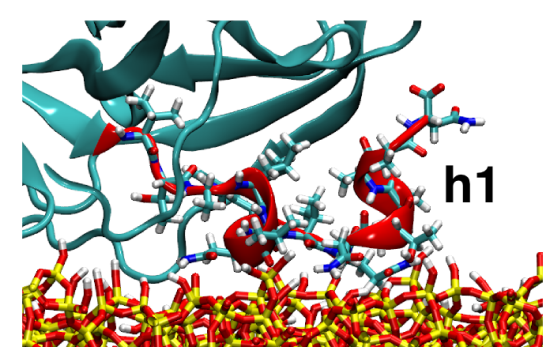

Figure 7: RESTmetaD free energy landscape of chymotrypsin during folding/unfolding of its $C$ terminal helical fragment $h_{1}$ in pure water (a) and adsorbed on amorphous silica (b), along with two representative snapshots of the respective most probable conformations $(c, d)$.

support the findings of our RESTmetaD simulation.

A different behavior is observed for the same sequence of residues embedded in its native environment, namely as C-terminal region of chymotrypsin. In this case, the free-energy profile predicted by RESTmetaD (Fig 7 (a)) does present a very well defined local minimum at helicity values around $80 \%$. In comparison, the native chymotrypsin structure, as taken from the Protein Data Bank and briefly equilibrated in TIP3P water, presents an $\mathrm{h}_{1}$ helicity of $70 \%$.

These simulations thus suggest that the protein environment around the C-terminal fragment $\mathrm{h}_{1}$ stabilizes it as an $\alpha$-helix, which would partially unfold if placed in pure water. The cause of this stabilization effect lies in the peculiar hydrophobic/hydrophilic matching between the side chains of the fragment and its neighbours, as shown in Fig. 8. In the folded conformation, the hydrophilic side chains of the helix point towards the solvent, while the hydrophobic ones are surrounded by the rest of the protein (Fig. 8(a)), which is composed, 
in turn, mostly by hydrophobic residues (Fig. 8(b)). The helix $\mathrm{h}_{1}$ is further stabilized by internal hydrogen bonds that connect the backbone's carbonyl and imide groups, while few hydrogen bonds are present with the rest of the protein (Fig. 8(c) and Fig. 9 (magenta)). Unfolding results in a loss of the internal hydrogen bonds and concurrent formation of some new hydrogen bonds with the rest of the protein (Fig. 8(d, e) and Fig. 9 (green)). However, the favourable enthalpic driving force associated with the formation of these additional hydrogen bonds when $h_{1}$ unfolds is not enough to counterbalance the loss of helix-internal bonds, resulting in a less stable conformation, in line with the predicted free energy profile (Fig. $7(\mathrm{a})$ ). Based on these results, we suggest that the helical stabilization of $\mathrm{h}_{1}$ is mainly due to an entropically-driven hydrophobic effect. The protein-fragment interactions are probably also responsible for the smoother shape of the profiles in comparison with the isolated fragment, where unfolding of each individual residue causes a small minimum in the computed profile (see Fig. 6).

Two facts are now worth recalling. First, in the most favourable orientation for adsorption on silica, chymotrypsin presents its helical fragments $h_{0}$ and $h_{1}$ towards the surface. $18 / 19$ Second, the hydrophilic side chains of $\mathrm{h}_{1}$ point outwards (Fig. 8(a)), providing good anchoring sites for stable surface adsorption ${ }^{18}$ (e.g. ASN236, GLN240, ASN245). The extent of unfolding induced by the $\mathrm{SiO}_{2}$ surface is now revealed with the help of RESTmetaD simulations. The free-energy profile as a function of $\mathrm{h}_{1}$ 's helicity in the presence of $\mathrm{SiO}_{2}$ is shown in Fig. 7(b). Compared with the situation in pure water (Fig. 7(a)), the surface does indeed lead to a flatter free energy profile. While the global minimum is still around $H=$ $75 \%$, a rugged local minimum region, separated by a barrier of about $20 \mathrm{~kJ} \mathrm{~mol}^{-1}$ and lying about $10 \mathrm{~kJ} \mathrm{~mol}^{-1}$ higher than the global minimum, is present around $H=17 \%$. Boltzmann integration of the profiles gives average helicity values of 77.7 and $68.9 \%$ in water and on the surface, respectively, at $300 \mathrm{~K}$. Therefore, we can safely conclude that the surface destabilizes the helical conformation of $\mathrm{h}_{1}$, but is not inducing a complete unfolding. This is in line with the experimental CD analysis, which reveals only a moderate decrease of the total 


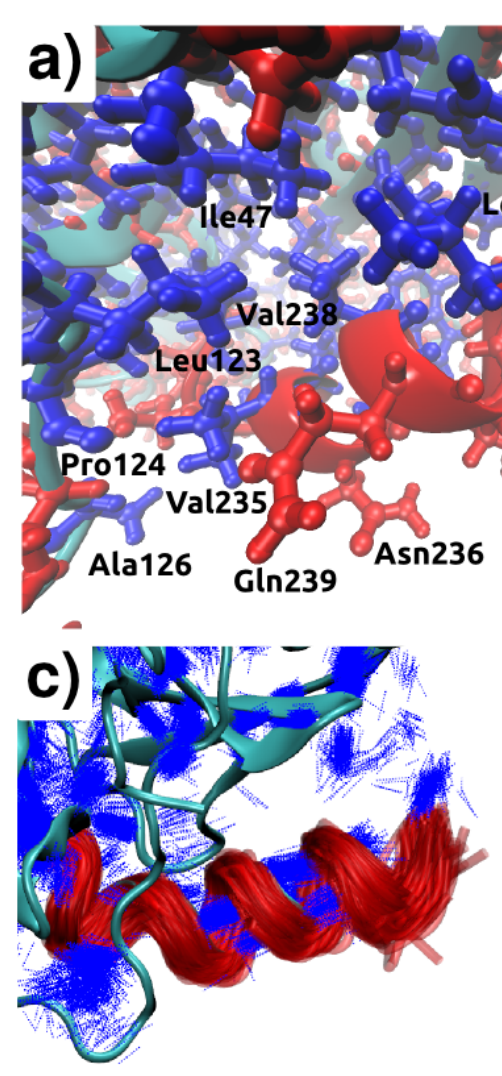

$92-100 \%$ helicity
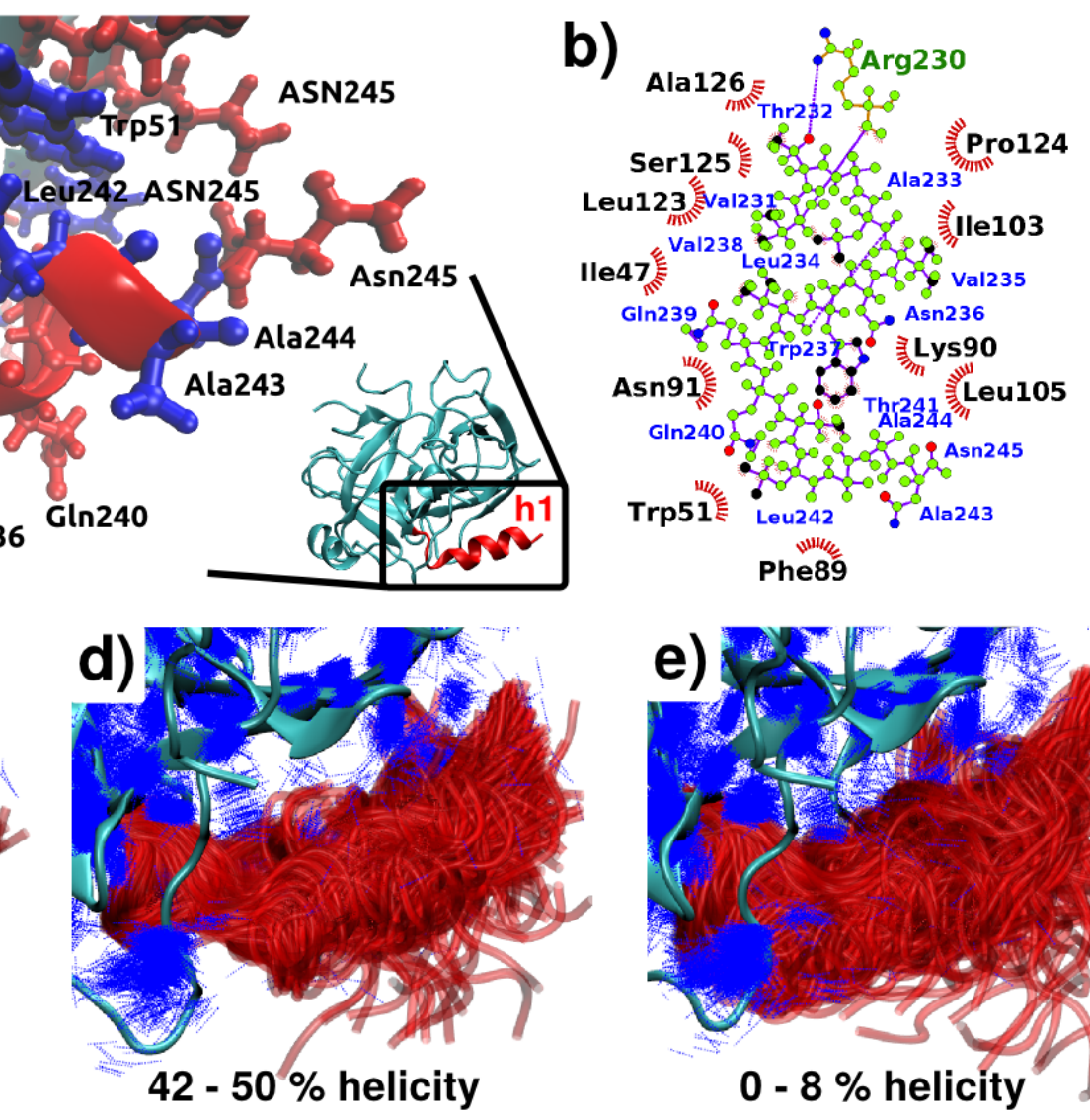

Figure 8: Stabilization of the helical conformation of the C-terminal $h_{1}$ fragment exerted by chymotrypsin due to matching of hydrophobic (blue) and hydrophilic (red) side chains (a). The protein residues (black labels) surrounding $h_{1}$ (blue labels, green atoms) are highlighted in the LigPlot ${ }^{80}$ interaction scheme in (b). Covalent bonds are shown as straight lines, hydrogen bonds as dashed lines, and hydrophobic interactions as red arcs. Panels (c,d,e) show superimposed structures of the

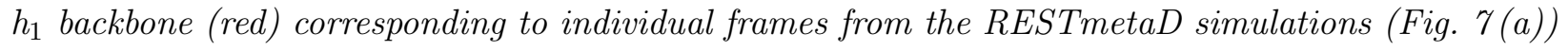
in three helicity windows (92-100, 42-50, 0-8 \%, respectively), highlighting the hydrogen bonds (thin blue lines) formed therein. A hydrogen bond is counted as present if the donor-acceptor distance is smaller than $3 \AA$ and the donor-H-acceptor angle lies between $160^{\circ}$ and $180^{\circ}$. 

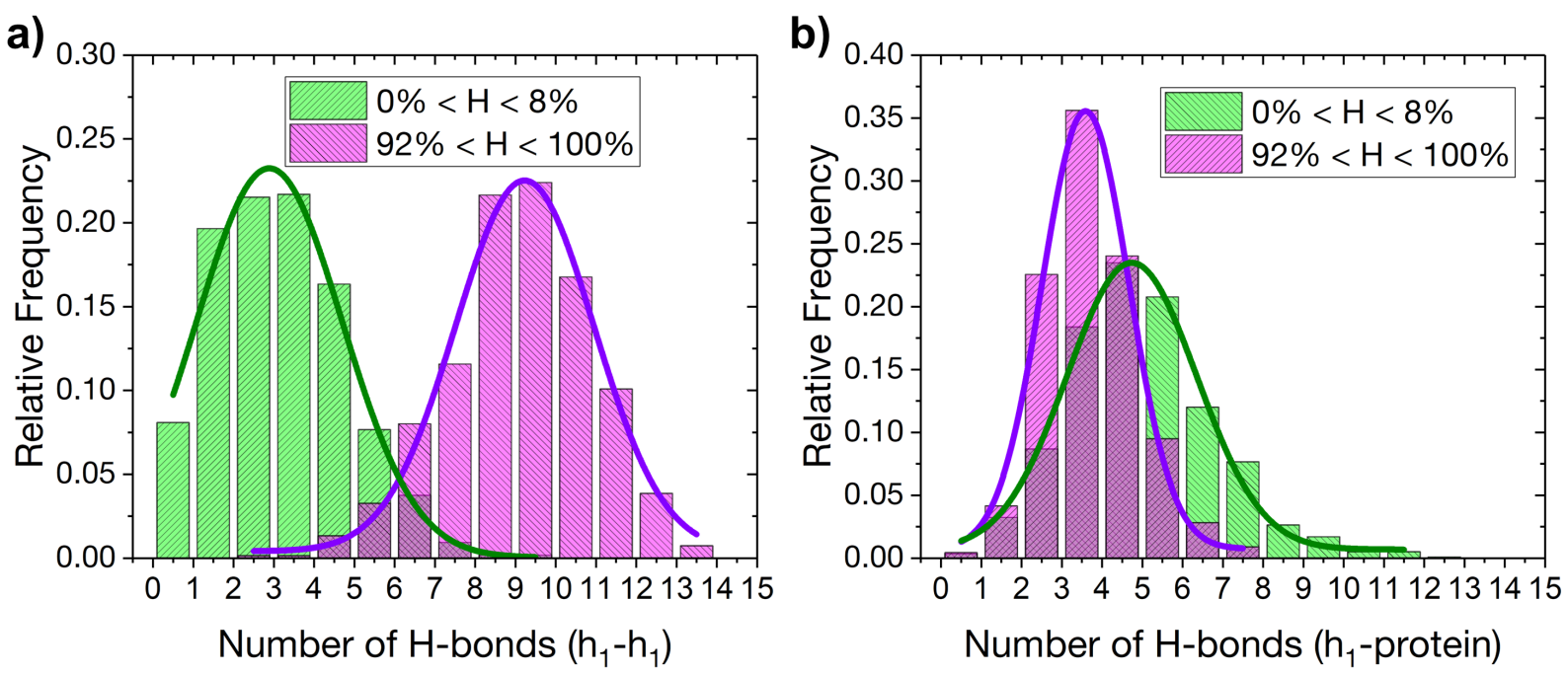

Figure 9: (a) Comparison between the distributions of the total number of internal hydrogen (H) bonds of the $h_{1}$ fragment, for low (green) and high (magenta) helicity. (b) Comparison between the distributions of the total number of $H$-bonds between residues in the $h_{1}$ fragment and the rest of the protein, for low (green) and high (magenta) helicity. Lines are Gaussian fittings to help the comparison. A hydrogen bond is counted as present if the donor-acceptor distance is smaller than $3 \AA$ and the donor-H-acceptor angle lies between $160^{\circ}$ and $180^{\circ}$.

chymotrypsin helicity (from $10 \%$ to $7.4 \%$ ) upon adsorption (Fig. 3).

\section{Structural cluster analysis}

The free-energy profiles presented above give an estimate of the expected helicity $H$ in the $\mathrm{h}_{1}$ fragment alone, as part of solvated chymotrypsin, and as part of the protein adsorbed on silica. To gain precise structural details about the conformations of the fragment in these three cases, we performed a cluster analysis in selected regions of the helicity collective variable (Fig. 10).

The isolated peptide, when unfolded (low $H$ values), is able to access many different conformations, which results in the most-populated clusters containing only $15 \%$ to $33 \%$ of the total frames up to a helicity of $50 \%$. The dominant structure is hairpin-like to horseshoe-like (Fig. 10 (a), $H<33 \%$ ) before nucleation of a helix starts from the C-terminus of the fragment $(H=42-50 \%)$. At higher $H$ the helix further develops while the fragment straightens, the most-populated cluster now including $80 \%(H=67-75 \%)$ to all $(H=92-$ 

(green) and hydrophobic (grey) residues on opposite sides of the formed helix.

When the $\mathrm{h}_{1}$ fragment is part of chymotrypsin (Fig. 10(b)), at large $H$ values it assumes the same helical structure as in water. However, the constraints exerted by the rest of the protein both through the covalent attachment at the fragment's N-terminus and weaker protein-fragment interactions (see Fig. 8) limit the conformational variability also at lower $H$ values. This is evident both from the much larger percentage of frames in the mostpopulated clusters at $0 \%<H<50 \%$, and from the onset of helical formation starting earlier ( $H=25-33 \%$ ), this time from the bound N-terminus rather than the free C-terminus of the fragment. The overall structures are more straightened, again due to the protein's embedding environment.

The embedding constraint is even larger when chymotrypsin is adsorbed on silica (Fig. 10.(c)), leading to clusters containing $67 \%$ and $79 \%$ of all frames even at the lowest helicities. The conformation at helicities close to the secondary local free energy minimum of the corresponding profile (Fig. 7(b), $H=25-33 \%$ ) show a relatively large flexibility of the C terminus and a kinked, boomerang-like fragment structure with already some degree of hydrophobic/hydrophilic order.

The RMSD minimization and alignment of all structures in this analysis, however, limits our understanding about the affinity of the $\mathrm{h}_{1}$ for the silica surface. For this reason, in Fig. 11 we show a superposition of all frames in the same helicity windows without any alignment or clustering. For clarity, only the different $\mathrm{h}_{1}$ fragment structures are actually superimposed, whereas the surface and the rest of the protein are drawn from a single ( $\mathrm{t}=0 \mathrm{~ns})$ frame, which best represents the embedding and constraining environment around the fragment.

At no value of helicity does the fragment firmly adsorb on the surface. It rather assumes both adsorbed and detached configurations. At the highest helicities, two main configurations (flat, adsorbed and tilted, desorbed) can be distinguished. At all lower helicities, including the regions around the global and local free-energy minima, the fragment is only partially 
adsorbed, with the C-terminus often being in close contact with the main protein's body. Anchoring to silica thus takes place via only few of the potentially available side-chain residues. Indeed, this is consistent with the chymotrypsin/silica anchoring sites revealed in our previous studies, $\frac{1819}{19}$ where the important anchoring role of also other protein regions, in particular the second helix $\mathrm{h}_{0}$, has been underlined.

\section{Discussion}

The combined results of experimental CD analysis and advanced-sampling MD simulations reveal that adsorption of chymotrypsin on silica surfaces leads to limited, but non-negligible, changes of its secondary structure. Most evident is a partial unfolding of the helical Cterminal fragment comprising the last 16 residues in the protein sequence, named $\mathrm{h}_{1}$ in this work. This fragment, in fact, partially unfolds when solvated in water as an isolated hexadecapeptide, because of its very flat free energy profile over the entire range of helical content (Fig. 6(a)). Being able to fold and unfold without significant energy penalty, it assumes, on average, a helicity of about $50 \%$, as revealed in CD experiments (Fig. 6(b)), suggested by structural de novo prediction servers, and quantified via RESTmetaD simulations. Its mostprobable unfolded conformations present a hairpin/horseshoe-like overall shape (Fig. 10 (a)). When folded, its hydrophilic and hydrophobic side chain residues become ordered at opposite sides of the nearly ideal $\alpha$-helical structure (Fig. 10 (a)).

This ordered hydropathy pattern is key for the observed stabilization of the folded helical conformation when $\mathrm{h}_{1}$ is embedded in the main protein's body, which provides a predominantly hydrophobic cleft in which $\mathrm{h}_{1}$ natively resides (Fig. 8). The entropic driving force of the classical hydrophobic effect makes the destabilization of the helical conformation energetically unfavourable, so that the free energy profile of $\mathrm{h}_{1}$ along the helicity coordinate presents a deep and well-defined minimum (Fig. 7(a)) at values close to an ideal helix. The average predicted helicity from the RESTmetaD simulations, $77.7 \%$, matches very well the 

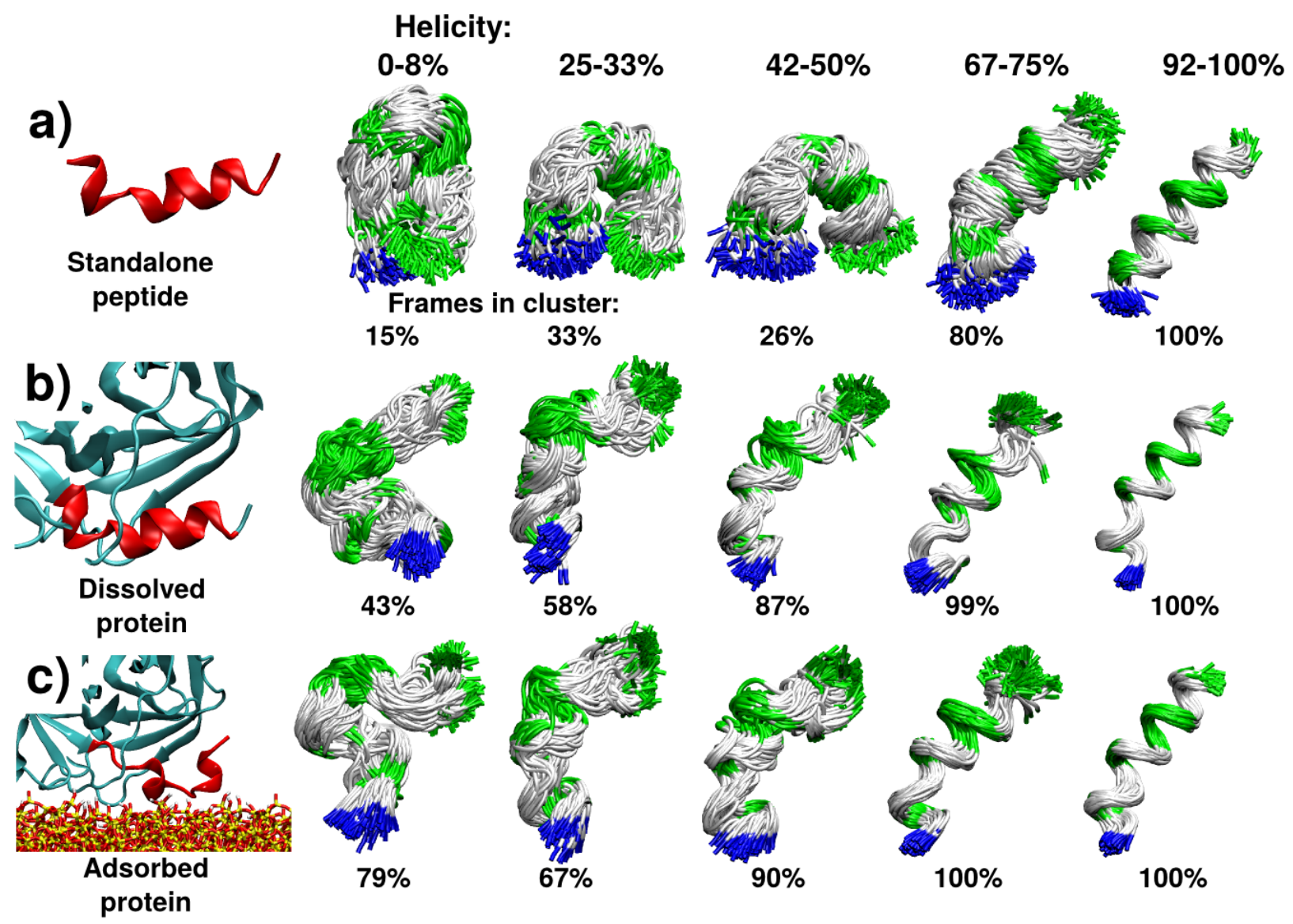

$79 \%$
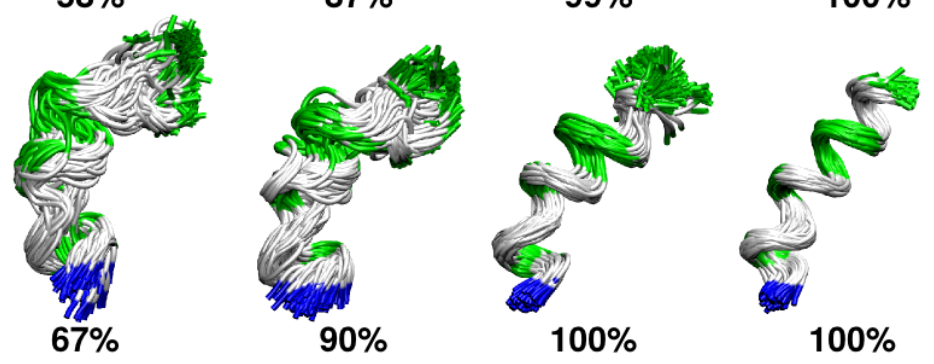

Figure 10: Representation of the most-populated clusters within $H$ intervals of 0-8, 25-33, 42-50, 67-75 and 92-100\% for the $h_{1}$ fragment as an isolated hexadecapeptide (a), as part of dissolved chymotrypsin (b), and as part of adsorbed chymotrypsin (c) (see snapshots on the left). The number and percentage of frames in the most populated clusters are reported underneath the corresponding superimposed frames. Hydrophilic residues are shown in green, hydrophobic in gray, the fragment's $N$-terminus in blue. 


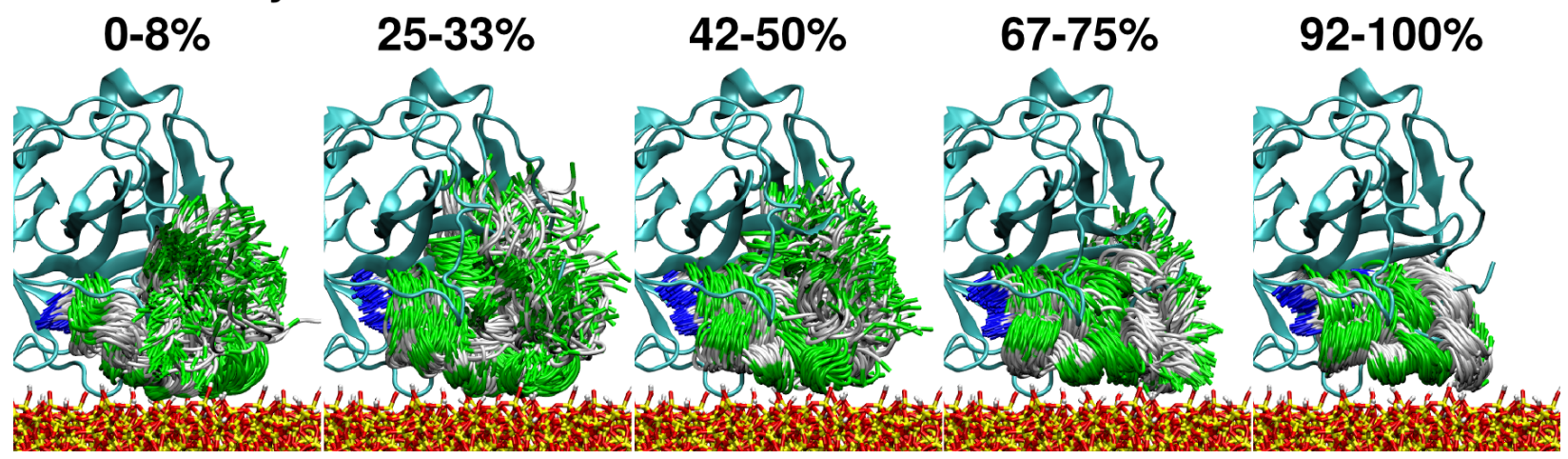

Figure 11: Superposition of all configurations of the $h_{1}$ fragment accessed during a RESTmetaD simulations of chymotrypsin adsorbed on silica (Fig. $7(b)$ ), in selected intervals of helicity $H$. The rest of the protein and the surface are represented with a single frame ( $t=0 \mathrm{~ns})$, as a reference. Colors of $h_{1}$ as in Fig. 10 .

helicity of $\mathrm{h}_{1}$ in the native PDB crystal structure $(70 \%)$.

When chymotrypsin adsorbs on silica, it does so in an orientation that brings both its

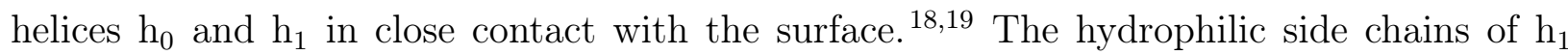
pointing outwards can now act as anchoring sites for the adsorbed protein, which causes a perturbation of both the native conformation of the helix and the aromatic couplings of neighbour Trp and Phe residues. This perturbation is not as strong as it would be required to unfold the helix completely, and in fact the $h_{1}$ fragment does not stably adsorb to the surface, even if unfolded (Fig. 11). However, the associated free-energy profile (Fig. 7(b)) is flatter than in solution, which suggests that a partial degree of destabilization is possible at $300 \mathrm{~K}$. This is consistent with the partial helicity loss and changes in the aromatic couplings estimated in our own experimental CD spectra of chymotrypsin adsorbed on silica nanoparticles (Fig. $3(\mathrm{a})$ ), as well as in a previous work. 20

Besides these effects, the CD spectra indicate only very minor changes of the protein's secondary structure. The stability of the globular tertiary structure of chymotrypsin is indeed due to the large extent of $\beta$-sheet elements in its interior. Since these are hardly perturbed after adsorption, we can infer that the overall tertiary structure of the protein remains essentially the same, which is in line with suggestions of previous studies. $\frac{21}{2 n d i r e c t l y, ~ t h i s ~}$ 
conclusion is supported by the much more evident spectral changes measured with CD upon heating of chymotrypsin up to $65^{\circ} \mathrm{C}$, promoting irreversible structural changes (Fig. 3(b) and Fig.S1 $(\mathrm{g}))$.

Adsorption and heating have different effects on the ellipticity band at $205 \mathrm{~nm}$, characteristic of $\pi \rightarrow \pi^{*}$ transitions in the peptide bond. The minimum shifts to larger wavelengths and decreases its intensity in the first case, while it shifts to smaller wavelengths and increases its intensity in the second case. In both cases, however, the secondary minimum at $230 \mathrm{~nm}$ evidently disappears. The disappearance of this minimum might be consistent with changes in the aromatic contributions both upon adsorption and heating, as qualitatively observed in our standard MD simulations at high temperature (Fig. 4 and Fig.S4 in S.I.). A possible verification of this hypothesis could come from measurements of the near-UV CD spectrum between 260 and $310 \mathrm{~nm}$, which reflects purely the contribution of aromatic side-chains that are located in asymmetrical environment and is very sensitive to any conformational changes. $\frac{81}{1}$ The theoretically calculated spectra reproduce qualitatively well the shift of the $205 \mathrm{~nm}$ minimum as a consequence of selective unfolding of the $\mathrm{h}_{1}$ helix (Fig. 5).

It must be noted that both our experiments and our simulations present some limitations, so that interpretation of the results must be taken with care. In the experiments, partial desorption of previously adsorbed chymotrypsin may take place in the short time required to re-disperse the silica particles, so that the measured spectra may contain contribution from desorbed molecules. This means that the actual loss of helicity in the adsorbed state may be larger than estimated here. Also, precise determination of the protein concentrations, especially in the adsorbed state, is tricky and prone to errors. These lead to uncertainties in the conversion from raw CD signal to $\Theta_{M R E}$, and, in turn, in the quantification of secondary structural elements.

Additional contributions to the helicity loss could also be due to partial unfolding of the $\mathrm{h}_{0}$ helix, despite it being apparently much more stable than $\mathrm{h}_{1}$ (Fig. 4). These contributions are not considered in our RESTmetaD simulations, which are limited only to helicity 
variations in $\mathrm{h}_{1}$. Finally, the simulations themselves contain some degrees of uncertainty. First, the employed force fields neither include polarization effects, nor allow for proton exchanges between the protein and the surface after adsorption. Secondly, issues still remain with the accuracy of force field predictions of protein secondary structure components. This is particularly true for non-helical conformations, while the chosen parameter set has been shown to give reliable results for the helical content of proteins. ${ }^{82}$ Finally, the ergodicity of the conformational sampling is not guaranteed, not even under the strong biased provided by the combination of replica exchange with metadynamics. In fact, although all investigated systems explore the whole ranges of helicity well enough (as reported in Fig.S6 of the Supporting Information), the sampling of single replicas is not always ideally homogeneous (diffusive), as evident from the variation in the number of frames collected in equally large windows of helicities (see Fig. 10). The convergence of the free energy profiles is, on the contrary, rather good, as shown by their smooth evolution in Figs. 6(a) and 7), and by control simulations starting from different initial conditions (S.I., Fig.S2). They point, however, to the difficulties encountered when dealing with bulky protein systems rather than small oligopeptides. $\frac{29 \mid 36}{29}$

\section{Conclusions}

In conclusion, we have shown that prediction of structural changes that enzymes undergo upon adsorption on oxide surfaces can be rationalized at the atomic scale by means of a combination of an accurate structure-dependent spectroscopic technique, Circular Dichroism, with all-atom Molecular Dynamics simulations coupled with enhanced sampling methods targeting free energy. As part of this joint effort, methods to theoretically predict CD spectra from atomistic simulations were also compared and improved.

We have found that chymotrypsin loses part of its helical content, with minor perturbation of its overall tertiary structure, associated to changes in the aromatic interactions, after 


\section{Acknowledgement}

We acknowledge fruitful discussions with R. Meißner (TU Hamburg-Harburg, Germany) and Carole Perry (Nottingham Trent University, UK). This work was supported by the the Deutsche Forschungsgemeinschaft under grants KO 3811/3-1, WE 5837/1-1 and CO 1043/8-

1. Computational resources were provided by the North-German Supercomputing-Alliance (HLRN). JK and AM were supported by the National Research, Development and Innovation 
Office, Hungary under grants K120391, KH125597, and 2017-1.2.1-NKP-2017-00002. AM is a Bolyai János Research Fellow of the Hungarian Academy of Sciences.

\section{Supporting Information Available}

Supporting Information includes: sensitivity of the conformational ensemble of Chymotrypsin upon adsorption on $\mathrm{SiO}_{2}$ and heating; additional free energy profiles obtained from RESTmetaD simulations started from opposite initial conditions $(H=100 \%)$; extended analysis of conformational changes upon heating; snapshots to show the changes of the interactions of aromatic residues in proximity of the helical segments induced by temperature or with a variable constraint applied to the helicity; values of the CV (helicity) during the RESTmetaD simulation. This material is available free of charge via the Internet at http://pubs.acs.org/.

\section{References}

(1) Björnmalm, M.; Faria, M.; Caruso, F. Increasing the impact of materials in and beyond bio-nano science. J. Am. Chem. Soc. 2016, 138, 13449-13456, DOI: $10.1021 /$ jacs.6b08673.

(2) Wei, Q.; Becherer, T.; Angioletti-Uberti, S.; Dzubiella, J.; Wischke, C.; Neffe, A. T.; Lendlein, A.; Ballauff, M.; Haag, R. Protein interactions with polymer coatings and biomaterials. Angew. Chem., Int. Ed. 2014, 53, 8004-8031, DOI: 10.1002/anie.201400546.

(3) Davis, D. H.; Giannoulis, C. S.; Johnson, R. W.; Desai, T. A. Immobilization of RGD to 111 silicon surfaces for enhanced cell adhesion and proliferation. Biomaterials 2002, 23, 4019-4027, DOI: 10.1016/S0142-9612(02)00152-7.

(4) Service, R. F. Can Sensors Make a Home in the Body? Science 2002, 297, 962-963, DOI: $10.1126 /$ science.297.5583.962. 
(5) Voskerician, G.; Shive, M. S.; Shawgo, R. S.; Von Recum, H.; Anderson, J. M.; Cima, M. J.; Langer, R. Biocompatibility and biofouling of MEMS drug delivery devices. Biomaterials 2003, 24, 1959-1967, DOI: 10.1016/S0142-9612(02)00565-3.

(6) Kinch, M. S. An overview of FDA-approved biologics medicines. Drug Discovery Today 2015, 20, 393-398, DOI: 10.1016/j.drudis.2014.09.003.

(7) Weiss, W. F.; Young, T. M.; Roberts, C. J. Principles, approaches, and challenges for predicting protein aggregation rates and shelf life. J. Pharm. Sci. 2009, 98, 1246-1277, DOI: $10.1002 /$ jps.21521.

(8) Appendini, P.; Hotchkiss, J. H. Immobilization of lysozyme on food contact polymers as potential antimicrobial films. Packag. Technol. Sci. 1997, 10, 271-279, DOI: 10.1002/(SICI)1099-1522(199709/10)10:5<271::AID-PTS412>3.0.CO;2-R.

(9) Appendini, P.; Hotchkiss, J. H. Review of antimicrobial food packaging. Innovative Food Sci. Emerging Technol. 2002, 3, 113-126, DOI: 10.1016/S1466-8564(02)00012-7.

(10) Eu, B.; Cairns, A.; Ding, G.; Cao, X.; Wen, Z.-Q. Direct visualization of protein adsorption to primary containers by gold nanoparticles. J. Pharm. Sci. 2011, 100, 1663-1670, DOI: $10.1002 /$ jps.22410.

(11) Thevenot, P.; Hu, W.; Tang, L. Surface Chemistry Influence Implant Biocompatibility. Curr. Top. Med. Chem. 2008, 8, 270-280, DOI: 10.2174/156802608783790901.

(12) Higuchi, A.; Shirano, K.; Harashima, M.; Yoon, B. O.; Hara, M.; Hattori, M.; Imamura, K. Chemically modified polysulfone hollow fibers with vinylpyrrolidone having improved blood compatibility. Biomaterials 2002, 23, 2659-2666, DOI: 10.1016/s01429612(01)00406-9.

(13) MacDonald, D. E.; Deo, N.; Markovic, B.; Stranick, M.; Somasundaran, P. Adsorption and dissolution behavior of human plasma fibronectin on thermally and chem- 
ically modified titanium dioxide particles. Biomaterials 2002, 23, 1269-1279, DOI: 10.1016/s0142-9612(01)00317-9.

(14) Lu, D. R.; Park, K. Effect of surface hydrophobicity on the conformational changes of adsorbed fibrinogen. J. Colloid Interface Sci. 1991, 144, 271-281, DOI: 10.1016/00219797(91)90258-a.

(15) Collier, T. O.; Anderson, J. M. Protein and surface effects on monocyte and macrophage adhesion, maturation, and survival. J. Biomed. Mater. Res. 2002, 60, 487-496, DOI: $10.1002 / \mathrm{jbm} .10043$.

(16) Derr, L.; Dringen, R.; Treccani, L.; Hildebrand, N.; Colombi Ciacchi, L.; Rezwan, K. Physisorption of enzymatically active chymotrypsin on titania colloidal particles. J. Colloid Interface Sci. 2015, 455, 236-244, DOI: 10.1016/j.jcis.2015.05.022.

(17) Derr, L.; Steckbeck, S.; Dringen, R.; Colombi Ciacchi, L.; Treccani, L.; Rezwan, K. Assessment of the Proteolytic Activity of $\alpha$-Chymotrypsin Immobilized on Colloidal Particles by Matrix-Assisted Laser Desorption Ionization Time-of-Flight Mass Spectrometry. Anal. Lett. 2015, 48, 424-441, DOI: 10.1080/00032719.2014.951449.

(18) Hildebrand, N.; Köppen, S.; Derr, L.; Li, K.; Koleini, M.; Rezwan, K.; Colombi Ciacchi, L. Adsorption Orientation and Binding Motifs of Lysozyme and Chymotrypsin on Amorphous Silica. J. Phys. Chem. C 2015, 119, 7295-7307, DOI: $10.1021 /$ acs.jpcc.5b00560.

(19) Derr, L.; Hildebrand, N.; Köppen, S.; Kunze, S.; Treccani, L.; Dringen, R.; Rezwan, K.; Colombi Ciacchi, L. Physisorption of $\alpha$-chymotrypsin on $\mathrm{SiO} 2$ and TiO2: A comparative study via experiments and molecular dynamics simulations. Biointerphases 2016, 11, 011007, DOI: 10.1116/1.4940701.

(20) Zoungrana, T.; Findenegg, G. H.; Norde, W. Structure, Stability, and Activ- 
ity of Adsorbed Enzymes. J. Colloid Interface Sci. 1997, 190, 437-448, DOI: 10.1006/jcis. 1997.4895 .

(21) Welzel, P. B. Investigation of adsorption-induced structural changes of proteins at solid/liquid interfaces by differential scanning calorimetry. Thermochim. Acta 2002, 382, 175-188, DOI: 10.1016/S0040-6031(01)00728-6.

(22) Bolin, K. A.; Pitkeathly, M.; Miranker, A.; Smith, L. J.; Dobson, C. M. Insight into a Random Coil Conformation and an Isolated Helix: Structural and Dynamical Characterisation of the C-Helix Peptide from Hen Lysozyme. J. Mol. Biol. 1996, 261, 443-453, DOI: $10.1006 /$ jmbi.1996.0475.

(23) Cole, D. J.; Payne, M. C.; Colombi Ciacchi, L. Water structuring and collagen adsorption at hydrophilic and hydrophobic silicon surfaces. Phys. Chem. Chem. Phys. 2009, 11, 11395, DOI: 10.1039/b816125a.

(24) Montserret, R.; Aubert-Foucher, E.; McLeish, M. J.; Hill, J. M.; Ficheux, D.; Jaquinod, M.; van der Rest, M.; Deléage, G.; Penin, F. Structural Analysis of the Heparin-Binding Site of the NC1 Domain of Collagen XIV by CD and NMR. Biochemistry 1999, 38, 6479-6488, DOI: 10.1021/bi9900222.

(25) Long, J. R.; Oyler, N.; Drobny, G. P.; Stayton, P. S. Assembly of $\alpha$-helical Peptide Coatings on Hydrophobic Surfaces. J. Am. Chem. Soc. 2002, 124, 6297-6303, DOI: $10.1021 / \mathrm{ja0} 11624 \mathrm{n}$.

(26) Lundqvist, M.; Nygren, P.; Jonsson, B.-H.; Broo, K. Induction of Structure and Function in a Designed Peptide upon Adsorption on a Silica Nanoparticle. Angew. Chem. Int. Ed. 2006, 45, 8169-8173, DOI: 10.1002/anie.200600965.

(27) Nygren, P.; Lundqvist, M.; Broo, K.; Jonsson, B.-H. Fundamental Design Principles That Guide Induction of Helix upon Formation of Stable Peptide-Nanoparticle Complexes. Nano Lett. 2008, 8, 1844-1852, DOI: 10.1021/nl080386s. 
(28) Burkett, S. L.; Read, M. J. Adsorption-induced conformational changes of $\alpha$-helical peptides. Langmuir 2001, 17, 5059-5065, DOI: 10.1021/la010156s.

(29) Meißner, R. H.; Schneider, J.; Schiffels, P.; Colombi Ciacchi, L. Computational Prediction of Circular Dichroism Spectra and Quantification of Helicity Loss upon Peptide Adsorption on Silica. Langmuir 2014, 30, 3487-3494, DOI: 10.1021/la500285m.

(30) Sivaraman, B.; Fears, K. P.; Latour, R. A. Investigation of the effects of surface chemistry and solution concentration on the conformation of adsorbed proteins using an improved circular dichroism method. Langmuir 2009, 25, 3050-3056, DOI: $10.1021 / \operatorname{la} 8036814$.

(31) Wei, Y.; Thyparambil, A. A.; Latour, R. A. Protein helical structure determination using CD spectroscopy for solutions with strong background absorbance from 190 to 230 nm. Biochim. Biophys. Acta, Proteins Proteomics 2014, 1844, 2331-2337, DOI: 10.1016/j.bbapap.2014.10.001.

(32) Bernardi, R. C.; Melo, M. C.; Schulten, K. Enhanced sampling techniques in molecular dynamics simulations of biological systems. Biochim. Biophys. Acta, General Subjects 2015, 1850, 872-877, DOI: 10.1016/j.bbagen.2014.10.019.

(33) Wang, L.; Friesner, R. A.; Berne, B. J. Replica Exchange with Solute Scaling: a More Efficient Version of Replica Exchange with Solute Tempering (REST2). J. Phys. Chem. $B$ 2011, 115, 9431-9438, DOI: 10.1021/jp204407d.

(34) Barducci, A.; Bussi, G.; Parrinello, M. Well-Tempered Metadynamics: A Smoothly Converging and Tunable Free-Energy Method. Phys. Rev. Lett. 2008, 100, 020603, DOI: $10.1103 /$ physrevlett.100.020603.

(35) Bussi, G.; Gervasio, F. L.; Laio, A.; Parrinello, M. Free-Energy Landscape for $\beta$ Hairpin Folding from Combined Parallel Tempering and Metadynamics. J. Am. Chem. Soc. 2006, 128, 13435-13441, DOI: 10.1021/ja062463w. 
(36) Schneider, J.; Colombi Ciacchi, L. Specific Material Recognition by Small Peptides Mediated by the Interfacial Solvent Structure. J. Am. Chem. Soc. 2012, 134, 24072413, DOI: 10.1021/ja210744g.

(37) Bussi, G. Hamiltonian replica exchange in GROMACS: a flexible implementation. Mol. Phys. 2013, 112, 379-384, DOI: 10.1080/00268976.2013.824126.

(38) Barducci, A.; Bonomi, M.; Parrinello, M. Metadynamics. Wiley Interdiscip. Rev.: Comput. Mol. Sci. 2011, 1, 826-843, DOI: 10.1002/wcms.31.

(39) Bulheller, B. M.; Hirst, J. D. DichroCalc-circular and linear dichroism online. Bioinformatics 2009, 25, 539-540, DOI: 10.1093/bioinformatics/btp016.

(40) Micsonai, A.; Wien, F.; Kernya, L.; Lee, Y.-H.; Goto, Y.; Réfrégiers, M.; Kardos, J. Accurate secondary structure prediction and fold recognition for circular dichroism spectroscopy. Proc. Natl. Acad. Sci. 2015, 112, E3095-E3103, DOI: $10.1073 /$ pnas. 1500851112 .

(41) Anthis, N. J.; Clore, G. M. Sequence-specific determination of protein and peptide concentrations by absorbance at 205 nm. Protein Sci. 2013, 22, 851-858, DOI: $10.1002 /$ pro. 2253 .

(42) Kuipers, B. J.; Gruppen, H. Prediction of molar extinction coefficients of proteins and peptides using UV absorption of the constituent amino acids at $214 \mathrm{~nm}$ to enable quantitative reverse phase high-performance liquid chromatography- mass spectrometry analysis. J. Agric. Food Chem. 2007, 55, 5445-5451, DOI: 10.1021/jf070337l.

(43) Greenfield, N. J. Using circular dichroism spectra to estimate protein secondary structure. Nat. Protocols 2007, 1, 2876-2890, DOI: 10.1038/nprot.2006.202.

(44) Case, D.; Darden, T.; et al., T. C. AMBER 2015; 2015. 
(45) Cole, D. J.; Payne, M. C.; Csányi, G.; Mark Spearing, S.; Colombi Ciacchi, L. Development of a classical force field for the oxidized Si surface: Application to hydrophilic wafer bonding. J. Chem. Phys. 2007, 127, 204704, DOI: 10.1063/1.2799196.

(46) Sonnefeld, J.; Göbel, A.; Vogelsberger, W. Surface Charge Density on Spherical Silica Particles in Aqueous Alkali Chloride Solutions. Colloid Polym. Sci. 1995, 273, 926-931, DOI: $10.1007 /$ bf00660369.

(47) Dove, P. M.; Craven, C. M. Surface Charge Density on Silica in Alkali and Alkaline Earth Chloride Electrolyte Solutions. Geochim. Cosmochim. Acta 2005, 69, 4963-4970, DOI: $10.1016 /$ j.gca.2005.05.006.

(48) Kobayashi, M.; Juillerat, F.; Galletto, P.; Bowen, P.; Borkovec, M. Aggregation and Charging of Colloidal Silica Particles:âĂL' Effect of Particle Size. Langmuir 2005, 21, 5761-5769, DOI: 10.1021/la046829z.

(49) Legrand, A. P.; Grillet, Y.; Burneau, A.; Papirer, E.; Fubini, B. In The Surface Properties of Silicas; Legrand, A. P., Ed.; John Wiley and Sons, Inc., New York, 1998.

(50) Pronk, S.; Pall, S.; Schulz, R.; Larsson, P.; Bjelkmar, P.; Apostolov, R.; Shirts, M. R.; Smith, J. C.; Kasson, P. M.; van der Spoel, D.; et al., GROMACS 4.5: a HighThroughput and Highly Parallel Open Source Molecular Simulation Toolkit. Bioinformatics 2013, 29, 845-854, DOI: 10.1093/bioinformatics/btt055.

(51) Joung, I. S.; Cheatham, T. E. Determination of Alkali and Halide Monovalent Ion Parameters for Use in Explicitly Solvated Biomolecular Simulations. J. Phys. Chem. B 2008, 112, 9020-9041, DOI: 10.1021/jp8001614.

(52) Butenuth, A.; Moras, G.; Schneider, J.; Koleini, M.; Köppen, S.; Meißner, R.; Wright, L. B.; Walsh, T. R.; Colombi Ciacchi, L. Ab Initio Derived Force-Field Parameters for Molecular Dynamics Simulations of Deprotonated Amorphous-SiO2/Water Interfaces. Phys. Status Solidi B 2011, 249, 292-305, DOI: 10.1002/pssb.201100786. 
(53) Hess, B. P-LINCS: A Parallel Linear Constraint Solver for Molecular Simulation. J. Chem. Theory Comput. 2008, 4, 116-122, DOI: 10.1021/ct700200b.

(54) Bussi, G.; Donadio, D.; Parrinello, M. Canonical Sampling through Velocity Rescaling. J. Chem. Phys. 2007, 126, 014101, DOI: 10.1063/1.2408420.

(55) Humphrey, W.; Dalke, A.; Schulten, K. VMD: Visual Molecular Dynamics. J. Mol. Graph. 1996, 14, 33-38, DOI: 10.1016/0263-7855(96)00018-5.

(56) Affentranger, R.; Tavernelli, I.; Di Iorio, E. E. A novel Hamiltonian replica exchange MD protocol to enhance protein conformational space sampling. J. Chem. Theory. Comput. 2006, 2, 217-228, DOI: 10.1021/ct050250b.

(57) Rathore, N.; Chopra, M.; de Pablo, J. J. Optimal allocation of replicas in parallel tempering simulations. J. Chem. Phys. 2005, 122, 024111, DOI: 10.1063/1.1831273.

(58) Bonomi, M.; Branduardi, D.; Bussi, G.; Camilloni, C.; Provasi, D.; Raiteri, P.; Donadio, D.; Marinelli, F.; Pietrucci, F.; Broglia, R. A.; Parrinello, M. PLUMED: a portable plugin for free-energy calculations with molecular dynamics. Comput. Phys. Commun. 2009, 180, 1961-1972, DOI: 10.1016/j.cpc.2009.05.011.

(59) Tribello, G. A.; Bonomi, M.; Branduardi, D.; Camilloni, C.; Bussi, G. PLUMED 2 : New feathers for an old bird. Comput. Phys. Commun. 2014, 185, 604-613, DOI: 10.1016/j.cpc.2013.09.018.

(60) Gil-Ley, A.; Bussi, G. Enhanced Conformational Sampling Using Replica Exchange with Collective-Variable Tempering. J. Chem. Theory Comput. 2015, 11, 1077-1085, DOI: $10.1021 / \operatorname{ct} 5009087$.

(61) Daura, X.; Gademann, K.; Jaun, B.; Seebach, D.; van Gunsteren, W. F.; Mark, A. E. Peptide Folding: when Simulation Meets Experiment. Angew. Chem., 
Int. Ed. 1999, 38, 236-240, DOI: 10.1002/(sici)1521-3773(19990115)38:1/2<236::aidanie236>3.0.co;2-m.

(62) Bulheller, B. M.; Rodger, A.; Hirst, J. D. Circular and linear dichroism of proteins. Phys. Chem. Chem. Phys. 2007, 9, 2020-2035, DOI: 10.1039/B615870F.

(63) Woody, R. W.; Sreerama, N. Comment on "Improving protein circular dichroism calculations in the far-ultraviolet through reparametrizing the amide chromophore". J. Chem. Phys. 1999, 111, 2844-2845, DOI: 10.1063/1.479562.

(64) Besley, N. A.; Hirst, J. D. Theoretical Studies toward Quantitative Protein Circular Dichroism Calculations. J. Am. Chem. Soc. 1999, 121, 9636-9644, DOI: $10.1021 /$ ja9906271.

(65) Li, Z.; Robinson, D.; Hirst, J. D. Vibronic structure in the far-UV electronic circular dichroism spectra of proteins. Faraday Discuss. 2015, 177, 329-344, DOI: 10.1039/c4fd00163j.

(66) Kabsch, W.; Sander, C. Dictionary of protein secondary structure: pattern recognition of hydrogen-bonded and geometrical features. Biopolymers 1983, 22, 2577-2637, DOI: $10.1002 /$ bip.360221211.

(67) Thevenet, P.; Shen, Y.; Maupetit, J.; Guyon, F.; Derreumaux, P.; Tuffery, P. PEP-FOLD: an updated de novo structure prediction server for both linear and disulfide bonded cyclic peptides. Nucleic Acids Res. 2012, 40, W288-W293, DOI: $10.1093 /$ nar/gks419.

(68) Camproux, A.; Gautier, R.; Tufféry, P. A Hidden Markov Model Derived Structural Alphabet for Proteins. J. Mol. Biol. 2004, 339, 591-605, DOI: 10.1016/j.jmb.2004.04.005.

(69) Yang, J.; Yan, R.; Roy, A.; Xu, D.; Poisson, J.; Zhang, Y. The I-TASSER 
Suite: protein structure and function prediction. Nat. Methods 2014, 12, 7-8, DOI: 10.1038/nmeth.3213.

(70) Xu, D.; Zhang, Y. Ab initio protein structure assembly using continuous structure fragments and optimized knowledge-based force field. Proteins: Struct., Funct., Bioinf. 2012, 1715-1735, DOI: 10.1002/prot.24065.

(71) Holzwarth, G.; Doty, P. The ultraviolet circular dichroism of polypeptides1. J. Am. Chem. Soc. 1965, 87, 218-228, DOI: 10.1021/ja01080a015.

(72) Wu, J.; Yang, J. T.; Wu, C.-S. C. $\beta$-II conformation of all- $\beta$ proteins can be distinguished from unordered form by circular dichroism. Anal. Biochem. 1992, 200, 359-364, DOI: 10.1016/0003-2697(92)90479-Q.

(73) Grishina, I. B.; Woody, R. W. Contributions of tryptophan side chains to the circular dichroism of globular proteins: exciton couplets and coupled oscillators. Faraday Discuss. 1994, 99, 245-262, DOI: 10.1039/FD9949900245.

(74) Sasaki, T.; Kobayashi, M.; Kise, H. Active conformation of a-chymotrypsin in organic solvents as studied by circular dichroism. Biotechnol. Tech. 1997, 11, 387-390, DOI: 10.1023/A:1018456420407.

(75) Sola-Rabada, A.; Michaelis, M.; Oliver, D. J.; Roe, M. J.; Colombi Ciacchi, L.; Heinz, H.; Perry, C. C. Interactions at the Silica-Peptide Interface: Influence of the Extent of Functionalization on the Conformational Ensemble. Langmuir 2018, 34, 8255-8263, DOI: 10.1021/acs.langmuir.8b00874.

(76) Ferguson, N.; Day, R.; Johnson, C. M.; Allen, M. D.; Daggett, V.; Fersht, A. R. Simulation and Experiment at High Temperatures: Ultrafast Folding of a Thermophilic Protein by Nucleation-condensation. J. Mol. Biol. 2005, 347, 855-870, DOI: 10.1016/j.jmb.2004.12.061. 
(77) Heinig, M.; Frishman, D. STRIDE: a web server for secondary structure assignment from known atomic coordinates of proteins. Nucleic Acids Res. 2004, 32, W500-W502, DOI: $10.1093 /$ nar/gkh429.

(78) Liu, J.; Yu, G.; Zhou, J. Ribonuclease A adsorption onto charged self-assembled monolayers: A multiscale simulation study. Chem. Eng. Sci. 2015, 121, 331-339, DOI: 10.1016/j.ces.2014.07.021.

(79) Liu, J.; Peng, C.; Yu, G.; Zhou, J. Molecular simulation study of feruloyl esterase adsorption on charged surfaces: effects of surface charge density and ionic strength. Langmuir 2015, 31, 10751-10763, DOI: 10.1021/acs.langmuir.5b01491.

(80) Wallace, A. C.; Laskowski, R. A.; Thornton, J. M. LIGPLOT: a program to generate schematic diagrams of protein-ligand interactions. Protein Eng. Des. Sel. 1995, 8, 127134, DOI: $10.1093 /$ protein/8.2.127.

(81) Kardos, J.; Bódi, Á.; Závodszky, P.; Venekei, I.; Gráf, L. Disulfide-linked propeptides stabilize the structure of zymogen and mature pancreatic serine proteases. Biochemistry 1999, 38, 12248-12257, DOI: 10.1021/bi990764v.

(82) Maier, J. A.; Martinez, C.; Kasavajhala, K.; Wickstrom, L.; Hauser, K. E.; Simmerling, C. ff14SB: improving the accuracy of protein side chain and backbone parameters from ff99SB. J. Chem. Theory. Comput. 2015, 11, 3696-3713, DOI: 10.1021/acs.jctc.5b00255. 


\section{For Table of Contents Use Only}

Atomistic Details of Chymotrypsin Conformational Changes upon Adsorption on Silica

Nils Hildebrand, Monika Michaelis, Nina Wurzler, Zhuo Li, Jonathan D. Hirst, András Micsonai, József Kardos, Alejandro Gil-Ley, Giovanni Bussi, Susan Köppen, Massimo Delle Piane, Lucio Colombi Ciacchi

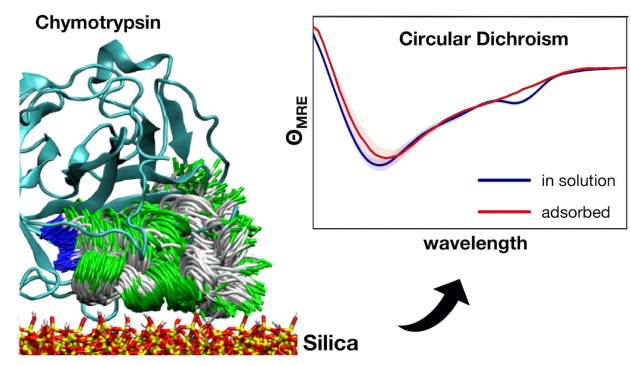

